\title{
On the Allen-Cahn/Cahn-Hilliard system with a geometrically linear elastic energy
}

\author{
Thomas Blesgen* and Anja Schlömerkemper ${ }^{\dagger}$
}

31 August 2012

\begin{abstract}
We present an extension of the Allen-Cahn/Cahn-Hilliard system which incorporates a geometrically linear ansatz for the elastic energy of the precipitates. The model contains both the elastic Allen-Cahn system and the elastic Cahn-Hilliard system as special cases and accounts for the microstructures on the microscopic scale. We prove the existence of weak solutions to the new model for a general class of energy functionals. We then give several examples of functionals that belong to this class. This includes the energy of geometrically linear elastic materials for $D<3$. Moreover we show this for $D=3$ in the setting of scalar-valued deformations, which corresponds to the case of anti-plane shear. All this is based on explicit formulas for relaxed energy functionals newly derived in this article for $D=1$ and $D=3$. In these cases we can also prove uniqueness of the weak solutions.
\end{abstract}

\section{Introduction}

In this article we study the Allen-Cahn/Cahn-Hilliard model (AC-CH model for short), which combines and extends two famous diffuse interface models: the Allen-Cahn equation and the Cahn-Hilliard equation. Both have been applied successfully to model segregation, precipitation and phase change phenomena in alloys and liquid mixtures in materials science, geology, physics, and biology, among others.

The AC-CH system was first introduced in [14, and its mathematical properties have been studied extensively, see, e.g., 3, 27, and references therein. We study here an extension of this model with a particular ansatz for the elastic energy.

The Allen-Cahn equation was first introduced without elasticity in 11; and the Cahn-Hilliard equation was first introduced without elasticity in [12. The Allen-Cahn equation is a second order partial differential equation of Ginzburg-Landau type for an unconserved orderparameter $b$ and thus can be used to model segregation and precipitation in solids, or other more general situations where a reordering of the crystal lattice occurs. Conversely, the

\footnotetext{
* Max Planck Institute for Mathematics in the Sciences, Inselstraße 22, D-04103 Leipzig, Germany, email: blesgen@is.mpg.de

†University of Würzburg, Institute for Mathematics, Emil-Fischer-Straße 40, D-97074 Würzburg, Germany, email: anja.schloemerkemper@mathematik.uni-wuerzburg.de
} 
Cahn-Hilliard equation is a fourth-order partial differential equation for a conserved order parameter $a$ striving to model phase change phenomena where one physical quantity like the volume of the phases is preserved. Throughout the paper we set $d=a+b$ as this plays a special role.

The Allen-Cahn system with linear elasticity was studied before in [9], the Cahn-Hilliard system with linear elasticity in [21, 29] and [13. An extension of the Cahn-Hilliard system with geometrically linear elasticity valid for single crystals was recently found in [7] for $D \leq 2$.

Except for the latter work, elastic effects due to small scale microstructures within the phases have been neglected. Here we treat the combined AC-CH model with elasticity in $D \leq 3$ dimensions. We state the new extended model in (1)-(3) in Section 2, This model provides a basis for the generalization of further existing isothermal diffuse interface models. Our definition of the extended model does not require any special assumptions (except regarding the regularity) on the stored energy functional $\mathcal{W}$. However, for the proof of existence of weak solutions, we require the Assumption $(\mathcal{A})$ phrased in Section 3 below.

We show that Assumption $(\mathcal{A})$ is satisfied in the following cases:

(i) Materials which follow the linear theory of elasticity developed by Eshelby [19] in the context of elastic inclusions and inhomogeneities (see a remark after Assumption $(\mathcal{A})$ );

(ii) materials in $D \leq 2$ which are well-described by a geometrically linear theory of elasticity (Theorem 5, for $D=2$, cf. also [10]);

(iii) materials in $D=3$ which are well-described by a geometrically linear theory of elasticity, where the deformations are assumed to be scalar-valued functions. This corresponds to the situation of anti-plane shear, see below for details and cf. Theorem 5 ,

The geometrically linear theory of elasticity allows for fine microstructures within the phases of the elastic materials taken into account. This becomes apparent in the explicit formulas for the relaxed energy functionals, which are, together with formulas for their derivatives, the basis of our proof of Theorem 5. In Section 4 we recall an explicit formula in $D=2$ derived by Chenchiah and Bhattacharya 15 . Moreover we prove explicit formulas for $D=1$, and for $D=3$ in the setting of scalar-valued deformations. (In [15] partial results for $D=3$ in the vectorial, i.e. the non-scalar, setting are shown.)

The coupling to elasticity changes significantly the morphology of the precipitates and the coarsening patterns, see, e.g., the classification in [20. For an intuitive picture of the microstructures taken into account we refer the reader to Figures 1 and 2 , Figure 1 displays exemplary macroscopic phases of a segregated material with phases $d$ being almost one (dark gray) and almost zero (light gray). In Figure 2 we show the corresponding microscopic length scale featuring microstructures; the left is a blow-up of a small region within the phase with $d$ being almost one and the right is a blow-up of a very small region within the phase with $d$ being almost zero. On this microscopic scale where we assume that it is sufficient to treat the elastic energies of single crystals, fine microstructures in the form of laminates occur, see, e.g., 30, 22.

The methods developed here apply generally to any established phase change and segregation model provided the temperature is conserved. (For non-isothermal settings, the validity of the second law of thermodynamics requires additional corrections which are not 


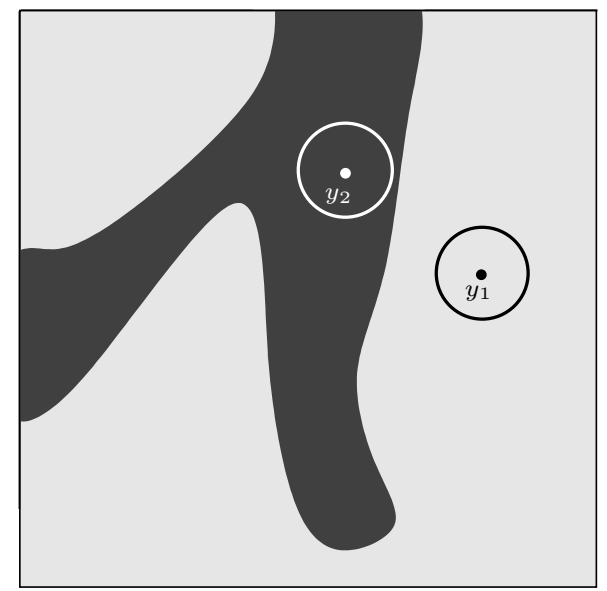

Figure 1: Macroscopic phases of a segregated material with phases $d$ being almost one (dark gray) and almost zero (light gray). In Figure 2 blow-ups of the circular regions are shown.
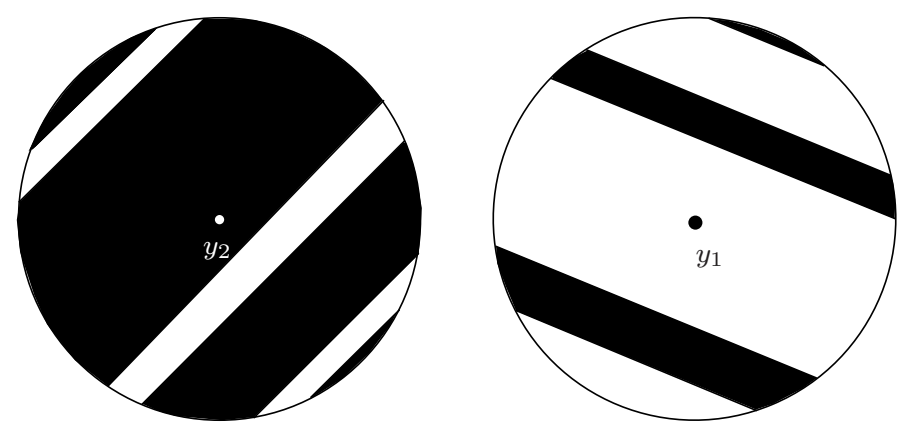

Figure 2: Microstructures with $d$ being almost one (left) and almost zero (right). The black part corresponds to $\tilde{d}=1$, while $\tilde{d}=0$ in the white regions. The average of $\tilde{d}$ equals $d$. 
studied here.) For further discussions of our model and the analytical results we refer to the conclusions in Section 6

\section{The AC-CH model and extensions}

Throughout this paper, let $\Omega \subset \mathbb{R}^{D}$ for $D \geq 1$ be a bounded domain with Lipschitz boundary which serves as an (unstressed) reference configuration. For a stop time $\mathcal{T}>0$, let $\Omega_{\mathcal{T}}:=$ $\Omega \times(0, \mathcal{T})$ denote the space-time cylinder. To the Allen-Cahn/Cahn-Hilliard system, first derived in [14, we add elasticity, possibly respecting the lamination microstructure, by introducing the system

$$
\begin{aligned}
\partial_{t} a & =\lambda \operatorname{div}\left(M(a, b) \nabla \frac{\partial F}{\partial a}\right), \\
\partial_{t} b & =-M(a, b) \frac{\partial F}{\partial b} \\
\mathbf{0} & =\operatorname{div}\left(\partial_{\varepsilon} \mathcal{W}(a+b, \varepsilon(\mathbf{u}))\right),
\end{aligned}
$$

where the function $a: \Omega_{\mathcal{T}} \rightarrow \mathbb{R}_{0}^{+}$is a conserved order parameter, typically a concentration, $b: \Omega_{\mathcal{T}} \rightarrow \mathbb{R}_{0}^{+}$is an unconserved order-parameter, specifying the reordering of the underlying lattice, $M(a, b)$ denotes the positive semi-definite mobility tensor, $\lambda>0$ is a small constant determining the interfacial thickness. The choice of $\mathcal{W}$ determines whether lamination microstructure occurs in the system, see (2.13).

By $\mathbf{u}: \Omega \rightarrow \mathbb{R}^{D}$ we describe the displacement field, such that a material point $x$ in the undeformed body $\Omega$ is at $x^{\prime}=x+\mathbf{u}(x)$ after the deformation. Then the (linearized) strain tensor is defined by

$$
\varepsilon(\mathbf{u}):=\frac{1}{2}\left(\nabla \mathbf{u}+\nabla \mathbf{u}^{t}\right),
$$

where $A^{t}$ denotes the transpose of a matrix $A \in \mathbb{R}^{D \times D}$. As usual, stands for the inner product in $\mathbb{R}^{D}$, that is $\mathbf{u} \cdot \mathbf{v}=\sum_{i=1}^{D} u_{i} v_{i}$, and for $A, B \in \mathbb{R}^{D \times D}$ we denote the inner product in $\mathbb{R}^{D \times D}$ by

$$
A: B:=\operatorname{tr}\left(A^{t} B\right)=\sum_{i, j=1}^{D} A_{i j} B_{i j} .
$$

Moreover, $|A|:=\sqrt{A: A}$ for $A \in \mathbb{R}^{D \times D}$ is the Frobenius norm. We denote the symmetric matrices in $\mathbb{R}^{D \times D}$ by $\mathbb{R}_{\mathrm{sym}}^{D \times D}$.

The functional $\mathcal{W}(a+b, \varepsilon(\mathbf{u}))$ represents the stored elastic energy density. We will choose it either according to the linear ansatz by Eshelby, $\mathcal{W}=W_{\text {lin }}$, or as a single crystal composite lamination energy, $\mathcal{W}=\widehat{W}$. The definition of $\widehat{W}$ is given in the coming section 2.1. The linear theory by Eshelby [19] developed in the context of elastic inclusions and inhomogeneities, can be summarized in the following ansatz for the elastic energy

$$
W_{\text {lin }}(d, \varepsilon):=\frac{1}{2}(\varepsilon-\underline{\varepsilon}(d)): C(d)(\varepsilon-\underline{\varepsilon}(d))
$$

for all $\varepsilon \in \mathbb{R}_{\mathrm{sym}}^{D \times D}, d:=a+b$, and $\underline{\varepsilon}(d):=d \underline{\varepsilon}$ with a constant $\underline{\varepsilon} \in \mathbb{R}_{\mathrm{sym}}^{D \times D}$. 
By $C(d)$ we denote the symmetric, positive definite and concentration-dependent elasticity tensor of the system that maps symmetric tensors in $\mathbb{R}^{D \times D}$ to themselves.

The system (10)-(3) is completed with the definition of the free energy

$$
F(a, b, \mathbf{u}):=\int_{\Omega} \psi(a, b)+\frac{\lambda}{2}\left(|\nabla a|^{2}+|\nabla b|^{2}\right)+\mathcal{W}(a+b, \varepsilon(\mathbf{u}))+W_{\text {ext }}(\varepsilon(\mathbf{u})) \mathrm{d} x,
$$

see [14, where $\psi(a, b)$ is the free energy density assumed to be

$$
\begin{aligned}
\psi(a, b) & :=\frac{\vartheta}{2}(g(a+b)+g(a-b))+\kappa_{1} a(1-a)-\kappa_{2} b^{2}, \\
g(s) & :=s \ln s+(1-s) \ln (1-s)
\end{aligned}
$$

for scalars $\kappa_{1}, \kappa_{2}>0$. The term $\frac{1}{2}[g(a+b)+g(a-b)]$ in (7) defines the entropic part of the free energy, given in the canonical Bernoulli form for perfect mixing, and $\vartheta>0$ is the constant temperature.

The functional $W_{\text {ext }}(\varepsilon)$ in (6) represents energy effects due to applied forces. In the absence of body forces, the work necessary to transform the undeformed body $\Omega$ into a state with displacement $\mathbf{u}$ is then

$$
-\int_{\partial \Omega} \mathbf{u} \cdot \sigma_{\text {ext }} \mathbf{n} \mathrm{d} S=-\int_{\Omega} \nabla \mathbf{u}: \sigma_{\text {ext }} \mathrm{d} x=-\int_{\Omega} \varepsilon(\mathbf{u}): \sigma_{\text {ext }} \mathrm{d} x,
$$

where we use that the applied stress $\sigma_{\text {ext }}$ is constant and symmetric. Consequently,

$$
W_{\text {ext }}(\varepsilon)=-\varepsilon: \sigma_{\text {ext }}
$$

is the energy density of the applied outer forces. The system (11)-(3) has to be solved in $\Omega_{\mathcal{T}}$ subject to the initial conditions

$$
a(t=0)=a_{0}, b(t=0)=b_{0} \quad \text { in } \Omega
$$

for given functions $a_{0}, b_{0}: \Omega \rightarrow \mathbb{R}$ subject to the Neumann boundary conditions for $a$, the no-flux boundary conditions, and the equilibrium condition for applied forces

$$
\nabla a \cdot \mathbf{n}=\nabla b \cdot \mathbf{n}=0, \quad J(a, b, \mathbf{u}) \cdot \mathbf{n}=0, \quad \sigma \cdot \mathbf{n}=\sigma_{\mathrm{ext}} \cdot \mathbf{n} \quad \text { on } \partial \Omega, t>0 .
$$

Here, $\sigma:=\partial_{\varepsilon} \mathcal{W}(a+b, \varepsilon(\mathbf{u}))$ defines the stress.

In (9), $\mathbf{n}$ is the unit outer normal to $\partial \Omega$. For simplicity, body forces are neglected and it is assumed that the boundary tractions are dead loads given by a constant symmetric tensor $\sigma_{\text {ext }}$. By $J$ we denote the mass flux, given by

$$
J(a, b, \mathbf{u}):=-M(a, b) \nabla \mu=-M(a, b) \nabla \frac{\partial F}{\partial a}(a, b, \mathbf{u}),
$$

with $\mu:=\frac{\partial F}{\partial a}$ the chemical potential.

The valid parameter range of $a$ and $b$ is, see Theorem 1 .

$$
0 \leq a+b \leq 1, \quad 0 \leq a-b \leq 1 .
$$


The inequalities are strict unless $(a, b)=(0,0)$ or $(a, b)=(1,0)$.

For $a_{0}=b_{0} \equiv 0$ in $\Omega$, we obtain the pathological solution $a=b \equiv 0$ in $\Omega_{\mathcal{T}}$. For $a_{0} \equiv 1$, $b_{0} \equiv 0$ in $\Omega$ we obtain a Cahn-Hilliard equation in $b$ and a pathological equation for $a$.

The system (11)-(3) includes as special case the elastic Cahn-Hilliard system (setting $b \equiv 0$, [21])

$$
\begin{aligned}
\partial_{t} a & =\lambda \operatorname{div}\left(M(a) \nabla \frac{\partial F}{\partial a}\right) \\
\mathbf{0} & =\operatorname{div}\left(\partial_{\varepsilon} \mathcal{W}(a, \varepsilon(\mathbf{u}))\right)
\end{aligned}
$$

with

$$
\begin{aligned}
F(a, \mathbf{u}) & :=\int_{\Omega} \psi(a)+\frac{\lambda}{2}|\nabla a|^{2}+\mathcal{W}(a, \varepsilon(\mathbf{u}))+W_{\text {ext }}(\varepsilon(\mathbf{u})) \mathrm{d} x, \\
\psi(a) & :=\frac{\vartheta}{2} a \ln a+(1-a) \ln (1-a)+\kappa_{1} a(1-a)
\end{aligned}
$$

and the boundary and initial conditions correspondingly to above. Moreover the system (11)-(3) includes as special case the elastic Allen-Cahn equations (setting $a \equiv \frac{1}{2}$, [9]) which for rescaled $b$ with $0<b<1$ read

$$
\begin{aligned}
\partial_{t} b & =-M(b) \frac{\partial F}{\partial b}, \\
\mathbf{0} & =\operatorname{div}\left(\partial_{\varepsilon} \mathcal{W}(b, \varepsilon(\mathbf{u}))\right),
\end{aligned}
$$

with

$$
\begin{aligned}
F(b, \mathbf{u}) & :=\int_{\Omega} \psi(b)+\frac{\lambda}{2}|\nabla b|^{2}+\mathcal{W}(b, \varepsilon(\mathbf{u}))+W_{\text {ext }}(\varepsilon(\mathbf{u})) \mathrm{d} x \\
\psi(b) & :=\frac{\vartheta}{2}(g(b)+g(1-b))+\frac{\kappa_{1}}{4}-\kappa_{2} b^{2}
\end{aligned}
$$

and $g$ as well as the boundary and initial conditions are as above. 
The system (11)-(3) is exemplary for an isothermal model that exhibits simultaneous ordering and phase transitions. Equation (11) is a diffusion law for $a$ governed by the flux $J$ and states the conservation of mass in $\Omega$. Equation (2) is a simple gradient flow in the descent direction $-\frac{\partial F}{\partial b}$. Equation (3) is a consequence of Newton's second law under the additional assumption that the acceleration $\partial_{t t} \mathbf{u}$ originally appearing on the left hand side can be neglected (this can be proved formally by a scaling argument and formally matched asymptotics). The vector equation (3) serves to determine the unknown displacement $\mathbf{u}$.

Remark 1. The equations (11)-(3) can be generalized to vector-valued mappings $a$, $b$. This allows to study situations with more than two phases present. To fix ideas and for the sake of a clear presentation, we restrict ourselves throughout this paper to scalars a and $b$.

Remark 2. The equations (1)-(3) with boundary conditions (9) and (6) and (7) comply with the second law of thermodynamics, which in case of isothermal conditions reads for a closed system

$$
\partial_{t} F(a(t), b(t), \mathbf{u}(t)) \leq 0
$$

This inequality can be verified by direct inspection similar to the calculations in [7].

In the proof of Theorem 2 we apply the following explicit formulation of (1)-(3) with constant mobility $M \equiv 1$

$$
\begin{aligned}
\partial_{t} a & =\lambda \triangle\left[\frac{\vartheta}{2}\left(g^{\prime}(a+b)+g^{\prime}(a-b)\right)+\kappa_{1}(1-2 a)+\frac{\partial \mathcal{W}}{\partial d}(a+b, \varepsilon(\mathbf{u}))-\triangle a\right], \\
\partial_{t} b & =\lambda \triangle b+\frac{\vartheta}{2}\left[g^{\prime}(a-b)-g^{\prime}(a+b)\right]+2 \kappa_{2} b-\frac{\partial \mathcal{W}}{\partial d}(a+b, \varepsilon(\mathbf{u})), \\
\mathbf{0} & =\operatorname{div}\left(\partial_{\varepsilon} \mathcal{W}(a+b, \varepsilon(\mathbf{u}))\right) .
\end{aligned}
$$

The subsequent section is devoted to the geometrically linear elastic energy for single crystals. This is a prerequisite to the discussion of existence and uniqueness results for the extensions of the Allen-Cahn/Cahn-Hilliard (AC-CH) model studied in Section 3

\subsection{The geometrically linear theory of elasticity in single crystals}

Our main objective in this subsection is to study a geometrically linear theory of elasticity in the context of isothermal phase transitions. For systematic reasons, we first recall the linear ansatz dating back to Eshelby, [19. As a byproduct of the existence theory proved in Section 3 we obtain a new existence result for the $\mathrm{AC}-\mathrm{CH}$ equations with linear elasticity. Subsequently, we introduce the geometrically linear elasticity theory that takes the laminates of the material into account.

In the following we assume that two phases are present in the considered material which may form microstructures as displayed, e.g., in Figures 3 and 4. We refer to the energy $W_{i}$, $i=1,2$ of each of the phases as microscopic energy, cf. (2.12), and to the energy $\widehat{W}(d, \varepsilon(\mathbf{u}))$ in (2.13), which reflects the effective behavior of the system with microstructures, as the macroscopic energy.

To determine the energy $\widehat{W}(d, \varepsilon(\mathbf{u}))$ in the geometrically linear theory we need to solve a local minimization problem, Eqn. (2.13) below, which we shall outline now. 
We assume that the volumes occupied by each of the two phases in $\Omega$ are measurable sets. In particular, if $\tilde{d}_{1} \equiv \tilde{d}, \tilde{d}_{2} \equiv 1-\tilde{d}$ characterize the two phases on the microscale, we have $\tilde{d}_{i} \in B V(\Omega ;\{0,1\})$ and $\tilde{d}_{1}+\tilde{d}_{2}=1$ a.e. in $\Omega$. The symbol $B V$ denotes the space of functions of bounded variation, see, e.g., 2, 31. By

$$
\langle\tilde{\varrho}\rangle:=f_{\Omega} \tilde{\varrho}(x) \mathrm{d} x:=\frac{1}{|\Omega|} \int_{\Omega} \tilde{\varrho}(x) \mathrm{d} x
$$

we denote the average of a function $\varrho$ in $\Omega$, where $|E|$ is the $D$-dimensional Lebesgue measure of a set $E$.

Let $\varepsilon_{i}^{T} \in \mathbb{R}_{\mathrm{sym}}^{D \times D}, i=1,2$, be the stress-free strain (or eigenstrain) of the $i$-th phase relative to the chosen reference configuration and $\alpha_{i}$ be its positive definite elasticity tensor. Then the elastic energy density of phase $i$ subject to a strain $\tilde{\varepsilon}$ is given by

$$
W_{i}(\tilde{\varepsilon}):=\frac{1}{2} \alpha_{i}\left(\tilde{\varepsilon}-\varepsilon_{i}^{T}\right):\left(\tilde{\varepsilon}-\varepsilon_{i}^{T}\right)+w_{i}
$$

for $w_{i} \geq 0$. In (2.12) we assumed for simplicity that $\alpha_{i}, \varepsilon_{i}^{T}$ and $w_{i}$ are constants, independent of $x \in \Omega$ and the order parameter $d$.

Under the assumption that the elastic energy adapts infinitely fast and that the surface energy between laminates of the microstructure can be neglected, the effective elastic energy is, [15,

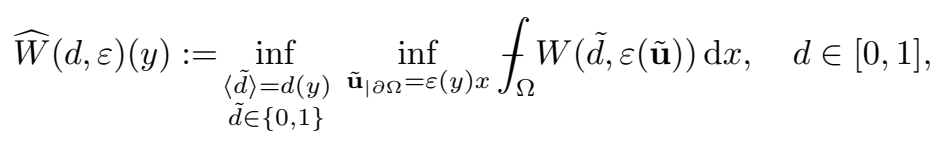

where we used

$$
W(\tilde{d}, \tilde{\varepsilon}):=\tilde{d} W_{1}(\tilde{\varepsilon})+(1-\tilde{d}) W_{2}(\tilde{\varepsilon}), \quad \tilde{d} \in\{0,1\} .
$$

The definition (2.13) requires further clarification. Firstly, $\tilde{\varepsilon}=\varepsilon(\tilde{\mathbf{u}}):=\frac{1}{2}\left(\nabla \tilde{\mathbf{u}}+\nabla \tilde{\mathbf{u}}^{t}\right)$, and instead of integrating over $\Omega$, one may integrate over $B_{r}(y)$, the open ball of radius $r$ around $y \in \Omega$, where the mean $\langle\cdot\rangle$ is now taken over $B_{r}(y)$. By homotopy arguments or by results in [18, any $r>0$ yields the same value of $\widehat{W}(d, \varepsilon)(y)$, as long as $B_{r}(y) \subset \Omega$. Taking the union of such balls then leads to (2.13). Secondly, the infimum over $\tilde{d}$ is the result of homogenization subject to the constraint that the volume fraction of the selected phase is preset by $d(y)$, see [17. Chapter 10]. This infimum is taken over functions $\tilde{d} \in B V(\Omega ;\{0,1\})$ as explained above ensuring $\widehat{W} \geq 0$. This is why (2.13) is only meaningful for $d \in[0,1]$. The second infimum is taken over functions $\tilde{\mathbf{u}} \in H^{1}\left(\Omega ; \mathbb{R}^{D}\right)$ where the condition $\tilde{\mathbf{u}}_{\mid \partial \Omega}=$ $\varepsilon(y) x$ has to be read as $\tilde{\mathbf{u}}(x)=\varepsilon(y) x$ for a.e. $x \in \partial \Omega$. This originates from the requirement that the functional $\widehat{W}$ thus defined must be quasi-convex, see [18, and is the result of relaxation theory, 18, 23, as follows. If for prescribed $d=a+b$ the microscopic elastic energy density is denoted by $W_{d}(\tilde{\varepsilon})$, then

$$
\widehat{W}_{d}(\varepsilon):=\inf _{\tilde{\mathbf{u}} \mid \partial \Omega=\varepsilon x} f_{\Omega} W_{d}(\varepsilon(\tilde{\mathbf{u}})) \mathrm{d} x
$$

is the elastic energy density of the material with macroscopic strain $\varepsilon$ after microstructure has formed. For $D=2$, explicit analytic formulas for $\widehat{W}$ are known, see [15] as well as formulas of the partial derivatives of $\widehat{W}$. These will be recalled in Section 4 . 


\section{Existence and uniqueness results for the AC-CH sys- tem}

The existence of solutions to the Allen-Cahn/Cahn-Hilliard equation without elasticity was studied in [11] with the help of a semigroup calculus. Existence and uniqueness of weak solutions to the Cahn-Hilliard equation with linear elasticity is proved in 21], with geometrically linear elasticity in [7]. Existence and uniqueness of weak solutions to the Allen-Cahn equation with linear elasticity is shown in 9 .

Subsequently we provide existence and uniqueness results for (1)-(3), where $\mathcal{W}$ is an elastic energy density satisfying the following assumption $(\mathcal{A})$.

$(\mathcal{A})$ The elastic energy density $\mathcal{W} \in C^{1}\left(\mathbb{R} \times \mathbb{R}_{\mathrm{sym}}^{D \times D} ; \mathbb{R}\right)$ satisfies the conditions

$(\mathcal{A} 1) \partial_{\varepsilon} \mathcal{W}(d, \cdot)$ is strongly monotone uniformly in $d$, i.e., there exists a constant $c_{1}>0$ such that for all $\varepsilon_{1}, \varepsilon_{2} \in \mathbb{R}_{\text {sym }}^{D \times D}$ and all $d \in \mathbb{R}$

$$
\left(\partial_{\varepsilon} \mathcal{W}\left(d, \varepsilon_{2}\right)-\partial_{\varepsilon} \mathcal{W}\left(d, \varepsilon_{1}\right)\right):\left(\varepsilon_{2}-\varepsilon_{1}\right) \geq c_{1}\left|\varepsilon_{2}-\varepsilon_{1}\right|^{2}
$$

$(\mathcal{A} 2)$ There exists a constant $C_{1}>0$ such that for all $d \in \mathbb{R}$ and all $\varepsilon \in \mathbb{R}_{\mathrm{sym}}^{D \times D}$

$$
\begin{aligned}
|\mathcal{W}(d, \varepsilon)| & \leq C_{1}\left(|d|^{2}+|\varepsilon|^{2}+1\right) \\
\left|\partial_{d} \mathcal{W}(d, \varepsilon)\right| & \leq C_{1}\left(|d|^{2}+|\varepsilon|^{2}+1\right) \\
\left|\partial_{\varepsilon} \mathcal{W}(d, \varepsilon)\right| & \leq C_{1}(|d|+|\varepsilon|+1)
\end{aligned}
$$

All constants in this article, unless explicitly stated otherwise, may depend on the material parameters $\alpha_{1}, \alpha_{2}, \varepsilon_{1}^{T}$ and $\varepsilon_{2}^{T}$, but are independent of $d$ and $\varepsilon$. Condition $(\mathcal{A} 1)$ states that $\mathcal{W}$ is convex in $\varepsilon$. The problem becomes non-convex through the dependence on $d$. One prominent example satisfying assumption $(\mathcal{A})$ is the elastic energy $W_{\text {lin }}$ in (5). In Section 5 we prove that also the relaxed energy functional $\widehat{W}$ defined in (2.13) satisfies the assumption $(\mathcal{A})$.

We require the condition

$$
\mathcal{W}\left(a_{0}+b_{0}, \varepsilon(\mathbf{u}(x, 0))\right)<\infty,
$$

where $\mathbf{u}(\cdot, 0)$ is the solution of (3) for $a=a_{0}, b=b_{0}$.

Theorem 1 (Existence of weak solutions). Let the mobility tensor $M$ be positive definite and continuous for all $a, b$ satisfying (10), $\mathcal{W}$ fulfill $(\mathcal{A}), \psi$ be given by (7) and the initial data $\left(a_{0}, b_{0}\right)$ satisfy (10) and (3.16). Then there exists a weak solution $(a, b, \mathbf{u})$ to (1) - (3) that satisfies

(i) $a, b \in C^{0, \frac{1}{4}}\left([0, \mathcal{T}] ; L^{2}(\Omega)\right)$,

(ii) $\partial_{t} a \in L^{2}\left(0, \mathcal{T} ; H^{1}(\Omega)^{*}\right), \partial_{t} b \in L^{2}\left(\Omega_{\mathcal{T}}\right)$,

(iii) $\mathbf{u} \in L^{\infty}\left(0, \mathcal{T} ; H^{1}\left(\Omega ; \mathbb{R}^{D}\right)\right)$,

(iv) The feasible parameter range of $(a, b)$ is given by (10).

Proof: The statements of the theorem can be proved with the methods developed in 9] for an Allen-Cahn system with linear elasticity and in 21] for a Cahn-Hilliard system with linear elasticity. We sketch the main steps. 
First we introduce the operator $\mathcal{M}$ associated to $w \mapsto-M \triangle w$ as a mapping from $H^{1}(\Omega)$ to its dual by

$$
\mathcal{M}(w) \eta:=\int_{\Omega} M \nabla w \cdot \nabla \eta \mathrm{d} x .
$$

From the Poincaré inequality and the Lax-Milgram theorem (which can be applied since $M$ is assumed to be positive definite) we know that $\mathcal{M}$ is invertible and we denote its inverse by $\mathcal{G}$, the Green's function. We have

$$
(M \nabla \mathcal{G} f, \nabla \eta)_{L^{2}}=\langle\eta, f\rangle \quad \text { for all } \eta \in H^{1}(\Omega), f \in\left(H^{1}(\Omega)\right)^{\prime} .
$$

For $f_{1}, f_{2} \in\left(H^{1}(\Omega)\right)^{\prime}$, we define the inner product

$$
\left(f_{1}, f_{2}\right)_{M}:=\left(M \nabla \mathcal{G} f_{1}, \nabla \mathcal{G} f_{2}\right)_{L^{2}}
$$

with the corresponding norm

$$
\|f\|_{M}:=\sqrt{(f, f)_{M}} \text { for } f \in\left(H^{1}(\Omega)\right)^{\prime} .
$$

For a small discrete step size $h>0$, chosen such that $\mathcal{T} h^{-1} \in \mathbb{N}$, for time steps $m \in \mathbb{N}$ with $0<m<\mathcal{T} h^{-1}$, and given values $a^{m-1}, b^{m-1} \in \mathbb{R}$, we introduce the discrete free energy functional

$$
F^{m, h}(a, b, \mathbf{u}):=F(a, b, \mathbf{u})+\frac{1}{2 h}\left\|a-a^{m-1}\right\|_{M}^{2}+\frac{1}{2 h}\left\|b-b^{m-1}\right\|_{L^{2}}^{2},
$$

where (in case of $m=1$ ) it holds $a^{0}=a_{0}, b^{0}=b_{0}$, the initial values of $a$ and $b$. By the direct method in the calculus of variations and Assumption $(\mathcal{A})$, it is possible to show that for $h$ sufficiently small, $F^{m, h}$ possesses a minimizer $\left(a^{m}, b^{m}, \mathbf{u}^{m}\right) \in H^{1}(\Omega) \times H^{1}(\Omega) \times H^{1}\left(\Omega ; \mathbb{R}^{D}\right)$. This minimizer solves the fully implicit time discretization of (1)-(3). Next the discrete solution is extended affine linearly to $(\bar{a}, \bar{b}, \overline{\mathbf{u}})$ by setting for $t=(\tau m+(1-\tau)(m-1)) h$ with suitable $\tau \in[0,1]$

$$
(\bar{a}, \bar{b}, \overline{\mathbf{u}})(t):=\tau\left(a^{m}, b^{m}, \mathbf{u}^{m}\right)+(1-\tau)\left(a^{m-1}, b^{m-1}, \mathbf{u}^{m-1}\right) .
$$

The validity of the second law of thermodynamics (cf. Remark 2) together with (3.16) implies that $F$ is non-increasing in time. In combination with a higher integrability condition on $\mathbf{u}$, 21, this allows to derive uniform estimates for $(\bar{a}, \bar{b}, \overline{\mathbf{u}})$. Compactness arguments then allow to pass to the limit $h \searrow 0$ and the limit solves (11)-(3).

In general, the uniqueness of solutions to (11)-(3) is open. However, we prove it in a special case for the linear elastic energy density $\mathcal{W}=W_{\text {lin }}$.

Theorem 2 (Uniqueness of solutions for linear elasticity). Let $\mathcal{W}=W_{\text {lin }}$ be given by (5), the material be homogeneous, i.e. the elasticity tensor $C$ be independent of $d$, and let $M \equiv 1$. Then the solution $(a, b, \mathbf{u})$ of Theorem 1 is unique in the spaces stated there.

Proof: The proof is very similar to the proof of uniqueness for the Cahn-Hilliard system [21] but we repeat it here because we later need to modify it. 
Fix $t_{0} \in(0, \mathcal{T})$. Let $\left(a^{k}, b^{k}, \mathbf{u}^{k}\right), k=1,2$ be two pairs of solutions to (2.1')-(2.3) and (5). The differences $a:=a^{1}-a^{2}, b:=b^{1}-b^{2}, \mathbf{u}:=\mathbf{u}^{1}-\mathbf{u}^{2}$ with corresponding difference of the chemical potentials $\mu:=\mu^{1}-\mu^{2}:=\frac{\partial F}{\partial a}\left(a^{1}, b^{1}\right)-\frac{\partial F}{\partial a}\left(a^{2}, b^{2}\right)$ solve the weak equations

$$
\begin{aligned}
& \int_{\Omega_{\mathcal{T}}}\left[-a \partial_{t} \xi+\lambda \nabla \mu \cdot \nabla \xi\right] \mathrm{d} x \mathrm{~d} t=0, \\
& \int_{\Omega_{\mathcal{T}}}\left[\partial_{t} b \eta+\lambda \nabla b \cdot \nabla \eta-\underline{\varepsilon}: C(\varepsilon(\mathbf{u})-(a+b) \underline{\varepsilon}) \eta\right] \mathrm{d} x \mathrm{~d} t \\
& =\int_{\Omega_{\mathcal{T}}}\left[\frac{\vartheta}{2}\left(g^{\prime}\left(a^{2}+b^{2}\right)-g^{\prime}\left(a^{1}+b^{1}\right)+g^{\prime}\left(a^{1}-b^{1}\right)-g^{\prime}\left(a^{2}-b^{2}\right)\right) \eta+2 \kappa_{2} b \eta\right] \mathrm{d} x \mathrm{~d} t, \\
& \int_{\Omega_{t_{0}}} C(\varepsilon(\mathbf{u})-\underline{\varepsilon}(a+b)): \varepsilon(\mathbf{u}) \mathrm{d} x \mathrm{~d} t=0
\end{aligned}
$$

for every $\xi, \eta \in L^{2}\left(0, \mathcal{T} ; H_{0}^{1}(\Omega)\right) \cap L^{\infty}\left(\Omega_{\mathcal{T}}\right)$ with $\partial_{t} \xi, \partial_{t} \eta \in L^{2}\left(\Omega_{\mathcal{T}}\right), \xi(\mathcal{T})=0$, where in order to get (3.21) we plugged in $\left(\mathbf{u}^{2}-\mathbf{u}^{1}\right) \mathcal{X}_{\left(0, t_{0}\right)}$ as a test function and integrated by parts. As a test function in (3.19) we pick

$$
\xi(x, t):= \begin{cases}\int_{t}^{t_{0}} \mu(x, s) d s, & \text { if } t \leq t_{0} \\ 0, & \text { if } t>t_{0}\end{cases}
$$

This shows

$$
\int_{\Omega_{t_{0}}} a \mu+\lambda \nabla(\mathcal{G} a) \cdot \nabla\left(\partial_{t} \mathcal{G} a\right) \mathrm{d} x \mathrm{~d} t=0 .
$$

The difference of the chemical potentials fulfils, with the help of (6),

$$
\begin{gathered}
\int_{\Omega_{\mathcal{T}}} \mu \zeta \mathrm{d} x \mathrm{~d} t=\int_{\Omega_{\mathcal{T}}}\left[\frac{\vartheta}{2}\left(g^{\prime}\left(a^{1}+b^{1}\right)-g^{\prime}\left(a^{2}+b^{2}\right)+g^{\prime}\left(a^{2}-b^{2}\right)-g^{\prime}\left(a^{1}-b^{1}\right)\right) \zeta\right. \\
\left.-2 \kappa_{1} a \zeta+\lambda \nabla a \cdot \nabla \zeta-\underline{\varepsilon}: C(\varepsilon(\mathbf{u})-(a+b) \underline{\varepsilon}) \zeta\right] \mathrm{d} x \mathrm{~d} t .
\end{gathered}
$$

We pick $\zeta:=\left(a^{1}-a^{2}\right) \mathcal{X}_{\left(0, t_{0}\right)}$. With (3.22) we obtain

$$
\begin{aligned}
& \frac{\lambda}{2}\left\|a\left(t_{0}\right)\right\|_{M}^{2}+\int_{\Omega_{t_{0}}} \lambda|\nabla a|^{2}-a \underline{\varepsilon}: C(\varepsilon(\mathbf{u})-\underline{\varepsilon}(a+b)) \mathrm{d} x \mathrm{~d} t \leq \\
& \quad \int_{\Omega_{t_{0}}} 2 \kappa_{1} a^{2}+\frac{\vartheta}{2}\left[\left|g^{\prime}\left(a^{1}+b^{1}\right)-g^{\prime}\left(a^{2}+b^{2}\right)\right|+\left|g^{\prime}\left(a^{1}-b^{1}\right)-g^{\prime}\left(a^{2}-b^{2}\right)\right|\right]|a| \mathrm{d} x \mathrm{~d} t .
\end{aligned}
$$

In (3.20) we choose $\eta:=\left(b^{1}-b^{2}\right) \mathcal{X}_{\left(0, t_{0}\right)}$ as a test function and add the resulting equation 
to (3.23) and use (3.21). We end up with

$$
\begin{aligned}
\frac{\lambda}{2}\left\|a\left(t_{0}\right)\right\|_{M}^{2}+ & \frac{1}{2}\left\|b\left(t_{0}\right)\right\|_{L^{2}}+\int_{\Omega_{t_{0}}}\left[\lambda\left(|\nabla a|^{2}+|\nabla b|^{2}\right)+\mathcal{W}(a+b, \varepsilon(\mathbf{u}))\right] \mathrm{d} x \mathrm{~d} t \\
\leq & \int_{\Omega_{t_{0}}} 2\left(\kappa_{1}|a|^{2}+\kappa_{2}|b|^{2}\right) \mathrm{d} x \mathrm{~d} t \\
& +\int_{\Omega_{t_{0}}} \frac{\vartheta}{2}\left[\left|g^{\prime}\left(a^{1}+b^{1}\right)-g^{\prime}\left(a^{2}+b^{2}\right)\right|+\left|g^{\prime}\left(a^{1}-b^{1}\right)-g^{\prime}\left(a^{2}-b^{2}\right)\right|\right](|a|+|b|) \mathrm{d} x \mathrm{~d} t .
\end{aligned}
$$

From Theorem 1 we know that the terms $g^{\prime}\left(a^{i} \pm b^{i}\right), i=1,2$ are finite, and $g^{\prime}$ is Lipschitz continuous. Applying first Young's inequality, then Gronwall's inequality, as $t_{0} \in(0, \mathcal{T})$ was arbitrary, we find $a=b=0$ in $\Omega_{\mathcal{T}}$. This finally yields

$$
\int_{\Omega_{\mathcal{T}}} \varepsilon(\mathbf{u}): C \varepsilon(\mathbf{u}) \mathrm{d} x \mathrm{~d} t=0 .
$$

With Korn's inequality this proves $\mathbf{u} \equiv 0$ in $\Omega_{\mathcal{T}}$.

\section{Explicit formulas for $\widehat{W}$}

In many situations like the numerical implementation of the extended models, the above definition (2.13) of $\widehat{W}$ is not practical since it is indirect and based on a local minimization. For these applications and for direct later use, we collect here some explicit formulas of the relaxed energy $\widehat{W}$ for $D \leq 2$ and for the scalar case in $D=3$.

\subsection{The case $D=2$}

As shown in [15], it holds

$$
\widehat{W}(d, \varepsilon)=d_{1} W_{1}\left(\varepsilon_{1}^{*}\right)+d_{2} W_{2}\left(\varepsilon_{2}^{*}\right)+\beta^{*} d_{1} d_{2} \operatorname{det}\left(\varepsilon_{2}^{*}-\varepsilon_{1}^{*}\right),
$$

where $\beta^{*}, \varepsilon_{1}^{*}$ and $\varepsilon_{2}^{*}$ are defined below. First we need to fix further notations following [15]. Let $\gamma^{*}>0$ be given by

$$
\gamma^{*}:=\min \left\{\gamma_{1}, \gamma_{2}\right\}
$$

where $\gamma_{i}$ is the reciprocal of the largest eigenvalue of $\alpha_{i}^{-1 / 2} T \alpha_{i}^{-1 / 2}, \alpha_{i}$ is the elastic modulus of laminate $i$, and the operator $T: \mathbb{R}_{\mathrm{sym}}^{2 \times 2} \rightarrow \mathbb{R}_{\mathrm{sym}}^{2 \times 2}$ is given by

$$
T \varepsilon=\varepsilon-\operatorname{tr}(\varepsilon) \mathrm{Id} .
$$

In [7] a recipe is given for the practical computation of $\gamma^{*}$. Here, we only remark that if the space groups of the two existing laminates are cubic, it holds

$$
\gamma^{*}=\min \left\{C_{1,11}-C_{1,12}, C_{2,11}-C_{2,12}, 2 C_{1,44}, 2 C_{2,44}\right\}
$$


The first subscript of $C$ denotes here the phase, the other two indices are the coefficients of the reduced elasticity tensor in Voigt notation, 28.

As shown in [15], the scalar $\beta^{*} \in\left[0, \gamma^{*}\right]$ determines the amount of translation of the laminates defined by

$$
\beta^{*}=\beta^{*}(d, \varepsilon):=\left\{\begin{array}{lll}
0 & \text { if } \varphi \equiv 0 & \text { (Regime 0), } \\
0 & \text { if } \varphi(0, d, \varepsilon)>0 & \text { (Regime I), } \\
\beta_{I I} & \text { if } \varphi(0, d, \varepsilon) \leq 0 \text { and } \varphi\left(\gamma^{*}, d, \varepsilon\right) \geq 0 & \text { (Regime II), } \\
\gamma^{*} & \text { if } \varphi\left(\gamma^{*}, d, \varepsilon\right)<0 & \text { (Regime III) }
\end{array}\right.
$$

In this definition, $\beta_{I I}=\beta_{I I}(d, \varepsilon)$ is the unique solution of $\varphi(\cdot, d, \varepsilon)=0$ with $\varphi$ defined by

$$
\begin{aligned}
\varphi(\beta, d, \varepsilon) & :=-\operatorname{det}\left(\triangle \varepsilon^{*}(\beta, d, \varepsilon)\right)=-\operatorname{det}\left[\alpha(\beta, d)^{-1} e(\varepsilon)\right], \\
\triangle \varepsilon^{*} & =\triangle \varepsilon^{*}(\beta, d, \varepsilon):=\varepsilon_{2}^{*}(\beta, d, \varepsilon)-\varepsilon_{1}^{*}(\beta, d, \varepsilon),
\end{aligned}
$$

and the yet undefined functions are specified below.

The four regimes have the following crystallographic interpretation, which follows from the construction of the optimal microstructure in the calculation of $\widehat{W}$.

Regime 0: The material is homogeneous and the energy does not depend on the microstructure. This occurs when $\alpha_{2}\left(\varepsilon_{2}^{T}-\varepsilon\right)-\alpha_{1}\left(\varepsilon_{1}^{T}-\varepsilon\right)=0$.

Regime I: There exist two optimal rank-I laminates.

This is characterized by

$$
\operatorname{det}\left[\left(d_{2} \alpha_{1}+d_{1} \alpha_{2}\right)^{-1}\left(\alpha_{2}\left(\varepsilon_{2}^{T}-\varepsilon\right)-\alpha_{1}\left(\varepsilon_{1}^{T}-\varepsilon\right)\right)\right]<0 .
$$

Regime II: The unique optimal microstructure is a rank-I laminate.

This regime occurs when the function

$$
\left[0, \gamma^{*}\right] \ni \beta \mapsto \operatorname{det}\left[\left(d_{2} \alpha_{1}+d_{1} \alpha_{2}-\beta T\right)^{-1}\left(\alpha_{2}\left(\varepsilon_{2}^{T}-\varepsilon\right)-\alpha_{1}\left(\varepsilon_{1}^{T}-\varepsilon\right)\right)\right]
$$

has a unique root (which we denote by $\beta_{I I}$ ).

Regime III: There exist two optimal rank-II laminates. This regime is present if the operator $\left(d_{2} \alpha_{1}+d_{1} \alpha_{2}-\gamma^{*} T\right)$ is invertible and

$$
\operatorname{det}\left[\left(d_{2} \alpha_{1}+d_{1} \alpha_{2}-\gamma^{*} T\right)^{-1}\left(\alpha_{2}\left(\varepsilon_{2}^{T}-\varepsilon\right)-\alpha_{1}\left(\varepsilon_{1}^{T}-\varepsilon\right)\right)\right]>0
$$

For illustration, we visualize prototypes of rank-I and rank-II laminates in Figures 3 and 4 .

To complete the definition (4.27), set

$$
\begin{aligned}
\alpha\left(\beta^{*}, d\right) & :=d_{2} \alpha_{1}+d_{1} \alpha_{2}-\beta^{*} T, \\
e(\varepsilon) & :=\alpha_{2}\left(\varepsilon_{2}^{T}-\varepsilon\right)-\alpha_{1}\left(\varepsilon_{1}^{T}-\varepsilon\right), \\
\varepsilon_{i}^{*} \equiv \varepsilon_{i}^{*}\left(\beta^{*}, d, \varepsilon\right) & :=\alpha^{-1}\left(\beta^{*}, d\right) e_{i}\left(\beta^{*}, d, \varepsilon\right), \\
e_{1}\left(\beta^{*}, d, \varepsilon\right) & :=\left(\alpha_{2}-\beta^{*} T\right) \varepsilon-d_{2}\left(\alpha_{2} \varepsilon_{2}^{T}-\alpha_{1} \varepsilon_{1}^{T}\right), \\
e_{2}\left(\beta^{*}, d, \varepsilon\right) & :=\left(\alpha_{1}-\beta^{*} T\right) \varepsilon+d_{1}\left(\alpha_{2} \varepsilon_{2}^{T}-\alpha_{1} \varepsilon_{1}^{T}\right) .
\end{aligned}
$$




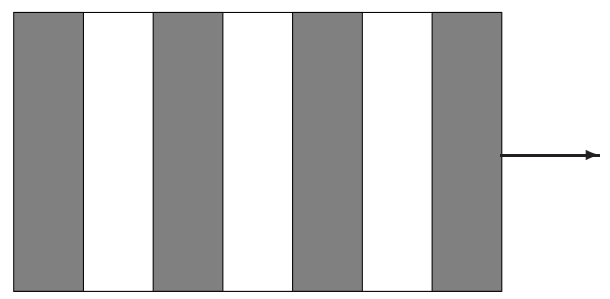

Figure 3: A two-phase rank-I laminate in two space dimensions with corresponding normal vector. The strains are constant in the shaded and in the unshaded regions. The volume fraction of both phases, 0.5 in the picture, is prescribed by the macroscopic parameter $d$.

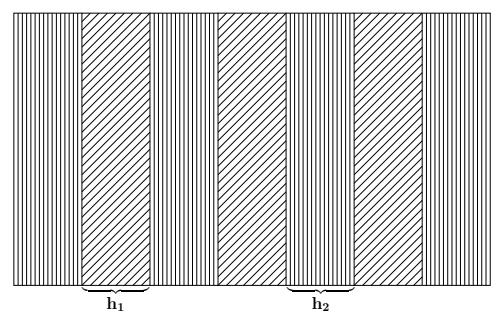

Figure 4: A two-phase rank-II laminate in two space dimensions. The widths $h_{1}$ and $h_{2}$ of the slabs should be much larger than the thickness of the layers between the slab.

Hence $\varepsilon_{2}^{*}-\varepsilon_{1}^{*}=\left[\alpha\left(\beta^{*}, d\right)\right]^{-1} e(\varepsilon)$.

Explicit computations of $\partial_{d} \widehat{W}$ and $\partial_{\varepsilon} \widehat{W}$ are lengthy. We recall the following results for the partial derivatives of $\widehat{W}$ in $D=2$ which are proved in [7]. Set

$$
\begin{aligned}
& \sigma_{i}^{*}:=\alpha_{i}\left(\varepsilon_{i}^{*}-\varepsilon_{i}^{T}\right), \\
& \bar{\sigma}^{*}:=d_{1} \sigma_{1}^{*}+d_{2} \sigma_{2}^{*} .
\end{aligned}
$$

Lemma 1. Let $D=2$ and let $\alpha_{i}$ and $T$ commute. Then

$$
\begin{aligned}
\frac{\partial \widehat{W}}{\partial \varepsilon}(d, \varepsilon)=d_{1} \alpha_{1}\left(\varepsilon_{1}^{*}\right. & \left.-\varepsilon_{1}^{T}\right)+d_{2} \alpha_{2}\left(\varepsilon_{2}^{*}-\varepsilon_{2}^{T}\right) \\
& + \begin{cases}\gamma^{*} d_{1} d_{2} \alpha^{-1}\left(\gamma^{*}, d\right)\left(\alpha_{1}-\alpha_{2}\right) T\left(\varepsilon_{2}^{*}-\varepsilon_{1}^{*}\right) & \text { in Regime III } \\
0 & \text { else. }\end{cases}
\end{aligned}
$$

Alternatively, in Regime III,

$$
\begin{aligned}
\frac{\partial \widehat{W}}{\partial \varepsilon}(d, \varepsilon)=d_{1}\left(\alpha_{2}-\gamma^{*} T\right) \alpha^{-1}\left(\gamma^{*}\right. & , d) \alpha_{1}\left(\varepsilon_{1}^{*}\left(\gamma^{*}, d, \varepsilon\right)-\varepsilon_{1}^{T}\right) \\
& +d_{2}\left(\alpha_{1}-\gamma^{*} T\right) \alpha^{-1}\left(\gamma^{*}, d\right) \alpha_{2}\left(\varepsilon_{2}^{*}\left(\gamma^{*}, d, \varepsilon\right)-\varepsilon_{2}^{T}\right) .
\end{aligned}
$$


Moreover it holds

$$
\begin{gathered}
\frac{\partial \widehat{W}}{\partial d}(d, \varepsilon)=\bar{\sigma}^{*}: \triangle \varepsilon^{*}+W_{1}\left(\varepsilon_{1}^{*}\right)-W_{2}\left(\varepsilon_{2}^{*}\right)+ \begin{cases}0 & \text { in Regimes } 0 \text { and I, } \\
\beta^{*} d_{1} d_{2} \frac{\partial \beta^{*}}{\partial d}\left\|\triangle \varepsilon^{*}\right\|^{2} & \text { in Regime II, } \\
\left(d_{1}-d_{2}\right) \gamma^{*} \varphi\left(\triangle \varepsilon^{*}\right) & \text { in Regime III. }\end{cases} \\
\frac{\partial \beta^{*}}{\partial d}= \begin{cases}\frac{\left(T\left(d_{2} \alpha_{1}+d_{1} \alpha_{2}-\beta T\right)^{-1}\left(\alpha_{2}-\alpha_{1}\right) \triangle \varepsilon^{*}\right): \triangle \varepsilon^{*}}{\left(\left(d_{2} \alpha_{1}+d_{1} \alpha_{2}-\beta T\right)^{-1}\left(T \triangle \varepsilon^{*}\right)\right): T \Delta \varepsilon^{*}} & \text { in Regime II, } \\
0 & \text { otherwise. }\end{cases} \\
\frac{\partial \beta^{*}}{\partial \varepsilon}= \begin{cases}\frac{1}{\left(\left(d_{2} \alpha_{1}+d_{1} \alpha_{2}-\beta T\right)^{-1}\left(T \triangle \varepsilon^{*}\right)\right): T \triangle \varepsilon^{*}}\left(\alpha_{1}-\alpha_{2}\right) \alpha^{-1} T \triangle \varepsilon^{*} & \text { in Regime II, } \\
0 & \text { otherwise. }\end{cases}
\end{gathered}
$$

\subsection{The scalar setting for $D=3$}

The scalar setting in three dimensions is characterized by the ansatz

$$
\mathbf{u}\left(x_{1}, x_{2}, x_{3}\right):=\left(\begin{array}{c}
x_{1} \\
x_{2} \\
\eta\left(x_{1}, x_{2}\right)
\end{array}\right)
$$

for the deformation, where $\eta$ is a scalar function (hence the name 'scalar' theory, see [5], [6]). Physically, (4.34) corresponds to anti-plane shear in the $x_{3}$-plane. Since

$$
\varepsilon(\mathbf{u})=\left(\begin{array}{ccc}
1 & 0 & \partial_{x_{1}} \eta \\
0 & 1 & \partial_{x_{2}} \eta \\
\partial_{x_{1}} \eta & \partial_{x_{2}} \eta & 0
\end{array}\right)
$$

$\nabla \eta$ determines the strain. This justifies to work with vectors $f=\nabla \eta \in \mathbb{R}^{2}$, not with matrices $\varepsilon(\mathbf{u})$. We replace (2.13) by

$$
\widehat{W}(d, f):=\inf _{\substack{\tilde{d} \tilde{\gamma}=d \\ \tilde{d} \in\{0,1\}}} \inf _{\tilde{\eta}_{\mid \partial \Omega^{\prime}}=f \cdot x} f_{\Omega^{\prime}} \tilde{d}_{1} W_{1}(\nabla \tilde{\eta})+\tilde{d}_{2} W_{2}(\nabla \tilde{\eta}) \mathrm{d} x, \quad d \in[0,1],
$$

where the domain of integration $\Omega^{\prime}$ is now two-dimensional. Therefore, $\tilde{d} \in B V\left(\Omega^{\prime} ;\{0,1\}\right)$, and it is well-understood from the context that

$$
\langle\tilde{d}\rangle:=\frac{1}{\left|\Omega^{\prime}\right|} \int_{\Omega^{\prime}} \tilde{d}(x) \mathrm{d} x
$$

denotes the two-dimensional average. In addition, in (4.35) we adapted the common notation for the scalar case and wrote $f$ instead of $\varepsilon$ for the strain. Finally, we set

$$
W_{i}(f):=\frac{1}{2} \alpha_{i}\left(f-f_{i}^{T}\right) \cdot\left(f-f_{i}^{T}\right)+w_{i}
$$


with $f_{i}^{T}$ the transformation strains in the scalar setting and $\alpha_{i}$ positive definite matrices.

In [15, partial results for $\widehat{W}$ in the non-scalar, i.e. vectorial setting are available for $D=3$. Yet, in general, the computation of $\partial_{d} \widehat{W}, \partial_{\varepsilon} \widehat{W}$ for $D=3$ as required for assumption $(\mathcal{A})$ remains currently open due to its complexity. This is the reason why we restrict ourselves to a special three-dimensional case. Our main result in this subsection is the following theorem that provides us with an explicit formula for $\widehat{W}$ in the scalar case.

Theorem 3 (Representation formula for $\widehat{W}$ and $D=3$ in the scalar setting). The functional $\widehat{W}$, given by (4.35), satisfies the explicit representation formula

$$
\widehat{W}(d, f)=d_{1} W_{1}\left(\nabla \eta_{1}^{*}\right)+d_{2} W_{2}\left(\nabla \eta_{2}^{*}\right)
$$

with

$$
\begin{aligned}
& \nabla \eta_{1}^{*}(d, f):=\left(d_{2} \alpha_{1}+d_{1} \alpha_{2}\right)^{-1}\left[\alpha_{2} f-d_{2}\left(\alpha_{2} f_{2}^{T}-\alpha_{1} f_{1}^{T}\right)\right] \\
& \nabla \eta_{2}^{*}(d, f):=\left(d_{2} \alpha_{1}+d_{1} \alpha_{2}\right)^{-1}\left[\alpha_{1} f+d_{1}\left(\alpha_{2} f_{2}^{T}-\alpha_{1} f_{1}^{T}\right)\right] .
\end{aligned}
$$

Theorem 3 states in particular that the scalar elastic theory in 3D is directly related to the non-scalar geometrically linear elasticity theory in 2D which was discussed in Subsection 4.1.

Proof: We apply the translation method, see for instance [16], 26] for an overview. In general, the function $\varphi$ which describes the translation is only quasi-convex. Here, as a benefit of the scalar theory, we can pick $\varphi$ as a convex function, cf. (4.42).

Starting from the inequality

$$
\varphi(f) \leq \inf _{\eta \mid \partial \Omega^{\prime}=f \cdot x} f_{\Omega^{\prime}} \varphi(\nabla \eta) \mathrm{d} x
$$

which is satisfied by any quasi-convex function $\varphi$, we obtain, similar to the reasoning in [15] for the non-scalar case in $D=2$ dimensions,

$$
\begin{gathered}
\widehat{W}(d, f) \geq \max _{\substack{\beta \geq 0 \\
W_{i}-\beta \varphi \text { convex }}} \min _{\substack{\tilde{d} \in B V\left(\Omega^{\prime} ;\{0,1\}\right) \\
\langle\tilde{d}\rangle=d}} \inf _{\eta \mid \partial \Omega^{\prime}=f \cdot x}\left[f_{\Omega^{\prime}} \tilde{d}_{1}\left(W_{1}-\beta \varphi\right)(\nabla \eta)+d_{2}\left(W_{2}-\beta \varphi\right)(\nabla \eta) \mathrm{d} x\right. \\
+\beta \varphi(f)]
\end{gathered}
$$

Consider

$$
\nabla \eta_{1}(\tilde{d}, \eta):=\frac{f_{\Omega^{\prime}} \tilde{d}_{1} \nabla \eta \mathrm{d} x}{f_{\Omega^{\prime}} \tilde{d}_{1} \mathrm{~d} x}, \quad \nabla \eta_{2}(\tilde{d}, \eta):=\frac{f_{\Omega^{\prime}} \tilde{d}_{2} \nabla \eta \mathrm{d} x}{f_{\Omega^{\prime}} \tilde{d}_{2} \mathrm{~d} x}
$$

Then

$$
d_{1} \nabla \eta_{1}(\tilde{d}, \eta)+d_{2} \nabla \eta_{2}(\tilde{d}, \eta)=f .
$$

Next we apply Jensen's inequality which is possible since $W_{i}-\beta \varphi$ is convex for $i=1,2$. Furthermore we possibly enlarge the set of admissible functions in the minimization over $\eta$ 
in (4.39) by considering the set of admissible functions $\nabla \eta_{1}, \nabla \eta_{2} \in \mathbb{R}^{2}$ with the constraint (4.41). Then (4.39) becomes

$$
\widehat{W}(d, f) \geq \max _{\substack{\beta \geq 0 \\ W_{i}-\beta \varphi \text { convex }}} \min _{\substack{\nabla \eta_{1}, \nabla \eta_{2} \in \mathbb{R}^{2} \\ d_{1} \nabla \eta_{1}+d_{2} \nabla \eta_{2}=f}}\left\{d_{1} W_{1}\left(\nabla \eta_{1}\right)+d_{2} W_{2}\left(\nabla \eta_{2}\right)+\beta \varphi(f)\right\} .
$$

If $D=2, \varphi$ is chosen to be $-\operatorname{det}(\varepsilon(\mathbf{u}))$. Here we set analogously

$$
\varphi(\nabla \eta):=\left(\partial_{x} \eta\right)^{2}+\left(\partial_{y} \eta\right)^{2} \geq 0
$$

Since $\varphi$ is quadratic, there exists a unique linear operator $T: \mathbb{R}^{2} \rightarrow \mathbb{R}^{2}$ such that $\varphi(f)=$ $\frac{1}{2} T f: f$, here simply $T f=2 f$. It holds

$$
\begin{aligned}
& \sum_{i=1}^{2} d_{i}\left(W_{i}-\beta \varphi\right)\left(\nabla \eta_{i}\right)+\beta \varphi(f) \\
&=\sum_{i=1}^{2} d_{i} W_{i}\left(\nabla \eta_{i}\right)+\beta[\underbrace{\varphi\left(d_{1} \nabla \eta_{1}+d_{2} \nabla \eta_{2}\right)-d_{1} \varphi\left(\nabla \eta_{1}\right)-d_{2} \varphi\left(\nabla \eta_{2}\right)}_{=: R}] .
\end{aligned}
$$

By a direct computation the remainder term $R$ can be rewritten,

$$
\begin{aligned}
2 R & =T\left(d_{1} \nabla \eta_{1}+d_{2} \nabla \eta_{2}\right):\left(d_{1} \nabla \eta_{1}+d_{2} \nabla \eta_{2}\right)-d_{1} T \nabla \eta_{1}: \nabla \eta_{1}-d_{2} T \nabla \eta_{2}: \nabla \eta_{2} \\
& =d_{1} d_{2}\left[-T \nabla \eta_{1}: \nabla \eta_{1}-T \nabla \eta_{2}: \nabla \eta_{2}+T \nabla \eta_{1}: \nabla \eta_{2}+T \nabla \eta_{2}: \nabla \eta_{1}\right] \\
& =-d_{1} d_{2} T\left(\nabla \eta_{2}-\nabla \eta_{1}\right):\left(\nabla \eta_{2}-\nabla \eta_{1}\right) \\
& =-2 d_{1} d_{2} \varphi\left(\nabla \eta_{2}-\nabla \eta_{1}\right) .
\end{aligned}
$$

So we have found

$$
\widehat{W}(d, f) \geq \max _{\substack{\beta \geq 0 \\ W_{i}-\beta \varphi \text { convex }}} \min _{\substack{\nabla \eta_{1}, \nabla \eta_{2} \in \mathbb{R}^{2} \\ d_{1} \nabla \eta_{1}+d_{2} \nabla \eta_{2}=f}}\left\{d_{1} W_{1}\left(\nabla \eta_{1}\right)+d_{2} W_{2}\left(\nabla \eta_{2}\right)-\beta d_{1} d_{2} \varphi\left(\nabla \eta_{2}-\nabla \eta_{1}\right)\right\} .
$$

Next we compute the optimal strains $\nabla \eta_{1}^{*}, \nabla \eta_{2}^{*}$. After differentiating the argument on the right in (4.43) for fixed $\beta$, we obtain

$$
\alpha_{1}\left(\nabla \eta_{1}^{*}-f_{1}^{T}\right)-\alpha_{2}\left(\nabla \eta_{2}^{*}-f_{2}^{T}\right)+\beta T\left(\nabla \eta_{2}^{*}-\nabla \eta_{1}^{*}\right)=0 .
$$

Using the constraint $d_{1} \nabla \eta_{1}^{*}+d_{2} \nabla \eta_{2}^{*}=f$, after rearrangement this yields the formulas

$$
\begin{aligned}
& \left(d_{1} \alpha_{2}+d_{2} \alpha_{1}-\beta T\right) \nabla \eta_{1}^{*}=\left(\alpha_{2}-\beta T\right) f-d_{2}\left(\alpha_{2} f_{2}^{T}-\alpha_{1} f_{1}^{T}\right), \\
& \left(d_{1} \alpha_{2}+d_{2} \alpha_{1}-\beta T\right) \nabla \eta_{2}^{*}=\left(\alpha_{1}-\beta T\right) f+d_{1}\left(\alpha_{2} f_{2}^{T}-\alpha_{1} f_{1}^{T}\right) .
\end{aligned}
$$

Setting $\beta=0$ (see below), this coincides with (4.37), (4.38).

The maximum over $\beta$ in (4.43) is attained at $\beta^{*}=0$ as $d_{1} d_{2} \varphi\left(\nabla \eta_{2}^{*}-\nabla \eta_{1}^{*}\right) \geq 0$. Hence, the translation is trivial in this setting. We obtain

$$
\widehat{W}(d, f) \geq d_{1} W_{1}\left(\nabla \eta_{1}^{*}\right)+d_{2} W_{2}\left(\nabla \eta_{2}^{*}\right)=: \widehat{W}_{-}(d, f) .
$$


It remains to estimate $\widehat{W}$ from above which amounts to showing that $\nabla \eta_{1}^{*}, \nabla \eta_{2}^{*}$ yield an optimal microstructure. Plugging in any microstructure $\tilde{d}$ with $\langle\tilde{d}\rangle=d$, from the definition (4.35) of $\widehat{W}$, we get the upper bound

$$
\widehat{W}(d, f) \leq \inf _{\tilde{\eta}_{\mid \partial \Omega^{\prime}}=f \cdot x} f_{\Omega^{\prime}} \tilde{d}_{1} W_{1}(\nabla \eta)+\tilde{d}_{2} W_{2}(\nabla \eta) \mathrm{d} x=: \widehat{W}_{+}(d, f) .
$$

As the domain of integration $\Omega^{\prime}$ in the scalar case with $D=3$ is two-dimensional, finding the optimal microstructure subsumes to the non-scalar case in $D=2$ dimensions. Hence, as can be seen from (4.26), $\beta^{*}=0$ occurs either for Regime 0 (no microstructure) or Regime I. The latter is equivalent to $\varphi\left(\varepsilon_{2}^{*}-\varepsilon_{1}^{*}\right)>0$. From a well-known argument, see [24, Lemma 4.1], this implies that $\varepsilon_{1}^{*}, \varepsilon_{2}^{*}$ are compatible. Besides this compatibility condition, any optimal microstructure must also satisfy the equilibrium condition

$$
[[\sigma]] \vec{n}=0
$$

which states that the jump of the stress in the normal direction of the laminate must vanish. Equation (4.46) originates from the Euler-Lagrange equation of the variational problem. Here, it is automatically satisfied since, due to (4.44), $[[\sigma]] \vec{n}=\sigma_{2}^{*}-\sigma_{1}^{*}=0$.

So there exists a unique rank-I lamination microstructure (unique up to a sign \pm 1 of direction of the laminate) that connects $\varepsilon_{1}^{*}$ and $\varepsilon_{2}^{*}$, and the strain of phase $i$ is $\nabla \eta_{i}^{*}, i=1,2$. This microstructure is optimal, $\widehat{W}_{+}(d, f)=\widehat{W}_{-}(d, f)$, which proves (4.36).

\subsection{The case $D=1$}

The methods of the previous subsection also apply to derive rigorously an explicit expression for $\widehat{W}$ in $D=1$. Here, quasi-convex functions are always convex, and the vector and scalar settings coincide. However, we use the notation in analogy to the vector case.

Theorem 4 (Representation formula for $\widehat{W}$ in $D=1$ ). For $D=1$, the functional $\widehat{W}$, given by (2.13), satisfies the explicit representation formula

$$
\widehat{W}(d, \varepsilon)=d W_{1}\left(\varepsilon_{1}^{*}\right)+(1-d) W_{2}\left(\varepsilon_{2}^{*}\right)
$$

with

$$
\begin{gathered}
\varepsilon_{1}^{*}(d, \varepsilon)=\frac{\alpha_{2}\left(\varepsilon-d_{2} \varepsilon_{2}^{T}\right)+d_{2} \alpha_{1} \varepsilon_{1}^{T}}{d_{2} \alpha_{1}+d_{1} \alpha_{2}}, \\
\varepsilon_{2}^{*}\left(d_{1}, \varepsilon\right)=\frac{\alpha_{1}\left(\varepsilon-d_{1} \varepsilon_{1}^{T}\right)+d_{1} \alpha_{2} \varepsilon_{2}^{T}}{d_{2} \alpha_{1}+d_{1} \alpha_{2}} .
\end{gathered}
$$

The partial derivatives of $\widehat{W}$ are given by

$$
\begin{aligned}
& \frac{\partial \widehat{W}}{\partial \varepsilon}(d, \varepsilon)=\frac{\alpha_{1} \alpha_{2}\left[d_{1}\left(\varepsilon-\varepsilon_{1}^{T}\right)+d_{2}\left(\varepsilon-\varepsilon_{2}^{T}\right)\right]}{d_{2} \alpha_{1}+d_{1} \alpha_{2}}, \\
& \begin{aligned}
\frac{\partial \widehat{W}}{\partial d}(d, \varepsilon)=W_{1}\left(\varepsilon_{1}^{*}\right)-W_{2}\left(\varepsilon_{2}^{*}\right)+\frac{\alpha_{1} \alpha_{2}}{\left(d_{2} \alpha_{1}+d_{1} \alpha_{2}\right)^{2}} & {\left[\left(\alpha_{1}-\alpha_{2}\right) \varepsilon^{2}+d_{1} \alpha_{1}\left(\varepsilon_{1}^{T}\right)^{2}-d_{2} \alpha_{2}\left(\varepsilon_{2}^{T}\right)^{2}\right.} \\
& +\left(\alpha_{2} \varepsilon_{2}^{T}-\alpha_{1} \varepsilon_{1}^{T}+\left(\alpha_{2}-\alpha_{1}\right)\left(d_{1} \varepsilon_{1}^{T}+d_{2} \varepsilon_{2}^{T}\right)\right) \varepsilon \\
& \left.+\left(d_{2} \alpha_{1}-d_{1} \alpha_{2}\right) \varepsilon_{1}^{T} \varepsilon_{2}^{T}\right] .
\end{aligned}
\end{aligned}
$$


Proof: We apply the same methods as in the proof of Theorem 3 ,

First, we estimate $W_{1}, W_{2}$ from below by their convexification. Similar to (4.43), we have for any convex function $\varphi$ and $\beta \geq 0$,

$$
\widehat{W}(d, \varepsilon) \geq \max _{\substack{\beta \geq 0 \\ W_{i}-\beta \varphi \text { convex }}} \min _{\substack{\varepsilon_{1}, \varepsilon_{2} \in \mathbb{R}^{D} \\ d \varepsilon_{1}+(1-d) \varepsilon_{2}=\varepsilon}}\left\{d\left(W_{1}-\beta \varphi\right)\left(\varepsilon_{1}\right)+(1-d)\left(W_{2}-\beta \varphi\right)\left(\varepsilon_{2}\right)+\beta \varphi(\varepsilon)\right\} .
$$

Secondly, we choose the optimal $\beta$ and $\varphi$. The later is an art when $D>1$. With arguments identical to the scalar case in $D=3$, it holds $\beta \equiv 0$, leaving us with the estimate

$$
\widehat{W}(d, \varepsilon) \geq \min _{\substack{\varepsilon_{1}, \varepsilon_{2} \in \mathbb{R} \\ d \varepsilon_{1}+(1-d) \varepsilon_{2}=\varepsilon}}\left\{d W_{1}\left(\varepsilon_{1}\right)+(1-d) W_{2}\left(\varepsilon_{2}\right)\right\}=: W_{-}(d, \varepsilon) .
$$

Next we compute the optimal strains $\varepsilon_{1}$, $\varepsilon_{2}$ on the right. For $d=1$, only $\varepsilon_{1}=\varepsilon$ is admissible and hence optimal. Now, let $0 \leq d<1$. We can then resolve the constraint by setting $\varepsilon_{2}=\frac{\varepsilon-d \varepsilon_{1}}{1-d}$ and need to calculate

$$
\min _{\varepsilon_{1} \in \mathbb{R}}\left\{d W_{1}\left(\varepsilon_{1}\right)+(1-d) W_{2}\left(\frac{\varepsilon-d \varepsilon_{1}}{1-d}\right)\right\} .
$$

After differentiation with respect to $\varepsilon_{1}$ and using (2.12) for the optimal $\varepsilon_{1}^{*}$ in (4.52), we are left with

$$
\alpha_{1}\left(\varepsilon_{1}^{*}-\varepsilon_{1}^{T}\right)=\alpha_{2}\left(\frac{\varepsilon-d \varepsilon_{1}^{*}}{1-d}-\varepsilon_{2}^{T}\right) .
$$

Rearrangement and resolution of $d \varepsilon_{1}^{*}+(1-d) \varepsilon_{2}^{*}=\varepsilon$ gives the formulas (4.48), (4.49) of the optimal strains. Since $\varepsilon_{1}^{*}(1, \varepsilon)=\varepsilon$, these formulas also state the correct solution when $d=1$.

In the final step, due to the definition of $\widehat{W}$, it holds

$$
\widehat{W}(d, \varepsilon) \leq \inf _{\mathbf{u} \mid \partial \Omega=\varepsilon x} f_{\Omega} \tilde{d} W_{1}(\varepsilon(\mathbf{u}))+(1-\tilde{d}) W_{2}(\varepsilon(\mathbf{u})) \mathrm{d} x=: W_{+}(d, \varepsilon)
$$

and $\tilde{d} \in B V(\Omega ;\{0,1\})$ may represent any microstructure with $\langle\tilde{d}\rangle=d$. The explicit construction of the optimal microstructure for which the upper bound and the lower bound coincide,

$$
W_{-}(d, \varepsilon)=\widehat{W}(d, \varepsilon)=W_{+}(d, \varepsilon),
$$

is again as in the proof of Theorem 3, This leads to (4.47).

Finally, the verification of (4.50), (4.51) follows from

$$
\frac{\partial \varepsilon_{1}^{*}}{\partial \varepsilon}(d, \varepsilon)=\frac{\alpha_{2}}{d_{2} \alpha_{1}+d_{1} \alpha_{2}}, \quad \frac{\partial \varepsilon_{2}^{*}}{\partial \varepsilon}(d, \varepsilon)=\frac{\alpha_{1}}{d_{2} \alpha_{1}+d_{1} \alpha_{2}}
$$

and

$$
\begin{aligned}
& \frac{\partial \varepsilon_{1}^{*}}{\partial d}=\frac{\alpha_{2}\left[\alpha_{2} \varepsilon_{2}^{T}-\alpha_{1} \varepsilon_{1}^{T}+\left(\alpha_{1}-\alpha_{2}\right) \varepsilon\right]}{\left(d_{2} \alpha_{1}+d_{1} \alpha_{2}\right)^{2}} \\
& \frac{\partial \varepsilon_{2}^{*}}{\partial d}=\frac{\alpha_{1}\left[\alpha_{2} \varepsilon_{2}^{T}-\alpha_{1} \varepsilon_{1}^{T}+\left(\alpha_{1}-\alpha_{2}\right) \varepsilon\right]}{\left(d_{2} \alpha_{1}+d_{1} \alpha_{2}\right)^{2}}
\end{aligned}
$$


which all can be verified by elementary computations.

Using the relationship $d_{1} \varepsilon_{1}^{*}+d_{2} \varepsilon_{2}^{*}=\varepsilon$ we find (4.50) and finally

$$
\begin{aligned}
\frac{\partial \widehat{W}}{\partial d}(d, \varepsilon)= & W_{1}\left(\varepsilon_{1}^{*}\right)-W_{2}\left(\varepsilon_{2}^{*}\right)+d_{1} W_{1}^{\prime}\left(\varepsilon_{1}^{*}\right) \frac{\partial \varepsilon_{1}^{*}}{\partial d}+d_{2} W_{2}^{\prime}\left(\varepsilon_{2}^{*}\right) \frac{\partial \varepsilon_{2}^{*}}{\partial d} \\
= & W_{1}\left(\varepsilon_{1}^{*}\right)-W_{2}\left(\varepsilon_{2}^{*}\right) \\
& +\frac{\alpha_{1} \alpha_{2}\left(\alpha_{2} \varepsilon_{2}^{T}-\alpha_{1} \varepsilon_{1}^{T}+\left(\alpha_{1}-\alpha_{2}\right) \varepsilon\right)\left[d_{1}\left(\varepsilon_{1}^{*}-\varepsilon_{1}^{T}\right)+d_{2}\left(\varepsilon_{2}^{*}-\varepsilon_{2}^{T}\right)\right]}{\left(d_{2} \alpha_{1}+d_{1} \alpha_{2}\right)^{2}} .
\end{aligned}
$$

Using

$$
d_{1}\left(\varepsilon_{1}^{*}-\varepsilon_{1}^{T}\right)+d_{2}\left(\varepsilon_{2}^{*}-\varepsilon_{2}^{T}\right)=\varepsilon-d_{2} \varepsilon_{2}^{T}-d_{1} \varepsilon_{1}^{T},
$$

this simplifies to identity (4.51).

\section{Existence results for the AC-CH system with mi- crostructural energy densities}

Above we have already remarked that the energy $W_{\text {lin }}$ satisfies assumption $(\mathcal{A})$ and thus Theorem 1 applies. In this section we investigate whether the results transfer to the geometrically linear theory of elasticity stated in the previous section.

The following statement is a consequence of Theorem 1

Theorem 5 (Existence of solutions for geometrically linear elastic energy). Let $\mathcal{W}=\widehat{W}$ be a function on $[0,1] \times \mathbb{R}_{\mathrm{sym}}^{D \times D}$. Assume that the mobility tensor $M$ be positive definite and continuous for all $a, b$ satisfying (10). Moreover, let $\psi$ be given by (7) and the initial data $\left(a_{0}, b_{0}\right)$ satisfy (10) and (3.16).

In $D=3$, let $\widehat{W}$ be given by 4.36 ) corresponding to the scalar setting. If $D=2$, let

(C 1) $\beta^{*}(d, \varepsilon)$ be independent of $\varepsilon$, and

(C2) $\alpha_{i}$ and $T$ commute whenever $\beta^{*} \in\left\{\gamma^{*}, \beta_{I I}\right\}$.

If $D=1$, let $\widehat{W}$ be given by (4.47). Then there exists a solution $(a, b, \mathbf{u})$ to (1)-(3) that satisfies

(i) $a, b \in C^{0, \frac{1}{4}}\left([0, \mathcal{T}] ; L^{2}(\Omega)\right)$,

(ii) $\partial_{t} a \in L^{2}\left(0, \mathcal{T} ; H^{1}(\Omega)^{*}\right), \partial_{t} b \in L^{2}\left(\Omega_{\mathcal{T}}\right)$,

(iii) $\mathbf{u} \in L^{\infty}\left(0, \mathcal{T} ; H^{1}\left(\Omega ; \mathbb{R}^{D}\right)\right)$,

(iv) The feasible parameter range of $(a, b)$ is given by (10).

Proof: We first consider the case $D<3$. The idea is to apply Theorem 1 . Therefore we

1. verify that $\widehat{W}$ satisfies assumption $(\mathcal{A})$ for $d \in[0,1]$,

2. extend $\widehat{W}$ to $\mathbb{R} \times \mathbb{R}_{\text {sym }}^{D \times D}$ in such a way that $(\mathcal{A})$ is fulfilled for all $d \in \mathbb{R}$. 
Then Theorem [1 can be applied and we obtain by part (iv) therein that effectively $d \in[0,1]$ in the system (1)-(3). Hence Theorem $[5$ follows with $\mathcal{W}=\widehat{W}$ as asserted.

We start with the second step and give the proofs of the former in dimensions $D=1$, $D=2$, and the scalar case in $D=3$ thereafter. We introduce the following extension $\widehat{W}_{0}(\cdot, \varepsilon): \mathbb{R} \rightarrow \mathbb{R}$ of $\widehat{W}(\cdot, \varepsilon)$ by

$$
\widehat{W}_{0}(d, \varepsilon):= \begin{cases}\widehat{W}(d, \varepsilon), & 0 \leq d \leq 1, \\ \varrho_{1}(d, \varepsilon), & 0>d>-1, \\ -d+1+\widehat{W}(0, \varepsilon), & d \leq-1 \\ \varrho_{2}(d, \varepsilon), & 1<d<2 \\ d-1+\widehat{W}(1, \varepsilon), & d \geq 2\end{cases}
$$

for functions $\varrho_{1}, \varrho_{2}$ that we may choose such that $\widehat{W}_{0}(\cdot, \cdot) \in C^{1}\left(\mathbb{R} \times \mathbb{R}_{\mathrm{sym}}^{D \times D}\right)$ and that $\widehat{W}_{0}$ fulfils $(\mathcal{A} 1)-(\mathcal{A} 2)$ for $d<0$ and $d>1$.

We further mention that the function $\widehat{W}_{0}$ defined in $(5.59)$ is positive and coercive for $|d| \rightarrow \infty$. We note that this construction is required for the first part of the proof of Theorem 1. where polynomial approximations of $\psi$ are constructed, where $d \notin[0,1]$ may occur.

It remains to show that $\widehat{W}$ fulfils $(\mathcal{A})$ for $d \in[0,1]$, which we prove independently for $D=1$, $D=2$, and the scalar case in $D=3$.

(i) Verification of $(\mathcal{A})$ for $D=1$ :

From (2.12), (4.47), (4.48) and (4.49), it follows $\widehat{W} \in C^{1}(\mathbb{R} \times \mathbb{R})$.

From (4.50), we obtain

$$
\left(\partial_{\varepsilon} \widehat{W}\left(d, \varepsilon_{2}\right)-\partial_{\varepsilon} \widehat{W}\left(d, \varepsilon_{1}\right)\right)\left(\varepsilon_{2}-\varepsilon_{1}\right)=\frac{\alpha_{1} \alpha_{2}}{d_{2} \alpha_{1}+d_{1} \alpha_{2}} \sum_{i=1}^{2} d_{i}\left(\varepsilon_{i}^{*}\left(d, \varepsilon_{2}\right)-\varepsilon_{i}^{*}\left(d, \varepsilon_{1}\right)\right)\left(\varepsilon_{2}-\varepsilon_{1}\right) .
$$

The condition ( $\mathcal{A} 1$ ), restricted to $d \in[0,1]$, follows, since by (4.48), (4.49) for $i=1,2$,

$$
\left(\varepsilon_{i}^{*}\left(d, \varepsilon_{2}\right)-\varepsilon_{i}^{*}\left(d, \varepsilon_{1}\right)\right)\left(\varepsilon_{2}-\varepsilon_{1}\right) \geq \frac{\min \left\{\alpha_{1}, \alpha_{2}\right\}}{\max \left\{\alpha_{1}, \alpha_{2}\right\}}\left|\varepsilon_{2}-\varepsilon_{1}\right|^{2},
$$

where $\frac{\min \left\{\alpha_{1}, \alpha_{2}\right\}}{\max \left\{\alpha_{1}, \alpha_{2}\right\}}>0$ by assumption. From (4.48), (4.49), for $i=1,2$ and $d \in[0,1]$ we have by similar arguments

$$
\left|\varepsilon_{i}^{*}(d, \varepsilon)\right| \leq c(|d|+|\varepsilon|+1) .
$$

This leads to $(\mathcal{A} 2)_{1}$, since by (4.47), (5.60),

$$
\begin{aligned}
|\widehat{W}(d, \varepsilon)| & \leq\left|W_{1}\left(\varepsilon_{1}^{*}\right)\right|+\left|W_{2}\left(\varepsilon_{2}^{*}\right)\right| \leq c\left(\left|\varepsilon_{1}^{*}\right|^{2}+\left|\varepsilon_{2}^{*}\right|^{2}+1\right) \\
& \leq c\left(|d|^{2}+|\varepsilon|^{2}+1\right) .
\end{aligned}
$$

For $d \in[0,1]$ we easily compute, for $i=1,2$,

$$
\left|\partial_{d} \varepsilon_{i}^{*}(d, \varepsilon)\right| \leq c(|\varepsilon|+1) .
$$

We use this estimate in (4.57), to obtain by (5.60)

$$
\begin{aligned}
\left|\partial_{d} \widehat{W}(d, \varepsilon)\right| & \leq c\left(\left|\varepsilon_{1}^{*}(d, \varepsilon)\right|^{2}+\left|\varepsilon_{2}^{*}(d, \varepsilon)\right|^{2}+1+\left(\left|\varepsilon_{1}^{*}(d, \varepsilon)\right|+\left|\varepsilon_{2}^{*}(d, \varepsilon)\right|\right)(|\varepsilon|+1)\right) \\
& \leq c\left(|d|^{2}+|\varepsilon|^{2}+1\right)
\end{aligned}
$$


which is $(\mathcal{A} 2)_{2}$ restricted to $d \in[0,1]$.

For $d \in[0,1]$ we derive with the help of (4.50), (4.58), (5.60)

$$
\begin{aligned}
\left|\partial_{\varepsilon} \widehat{W}(d, \varepsilon)\right| & \leq\left|\frac{\alpha_{1} \alpha_{2}}{\min \left\{\alpha_{1}, \alpha_{2}\right\}}\right|\left(\left|\varepsilon-\varepsilon_{1}^{T}\right|+\left|\varepsilon-\varepsilon_{2}^{T}\right|\right) \\
& \leq \max \left\{\alpha_{1}, \alpha_{2}\right\} c(|\varepsilon|+1) \\
& \leq c(|\varepsilon|+1),
\end{aligned}
$$

showing the validity of $(\mathcal{A} 2)_{3}$ restricted to $d \in[0,1]$.

(ii) Verification of $(\mathcal{A})$ for $D=2$ :

The regularity of $\widehat{W}$ required for $(\mathcal{A})$ follows directly from (4.24) and the related definitions. The constant $\gamma^{*}$ solely depends on $\alpha_{1}, \alpha_{2}$ since $\gamma^{*}=\min \left\{\gamma_{1}, \gamma_{2}\right\}$ and $\gamma_{i}$ is the reciprocal of the largest eigenvalue of $\alpha_{i}^{-1 / 2} T \alpha_{i}^{-1 / 2}$, see (4.25). Since $\beta^{*} \in\left[0, \gamma^{*}\right]$,

$$
\left|\beta^{*}(d, \varepsilon)\right| \leq c
$$

for a constant $c$ independent of $d$ and $\varepsilon$.

From (5.61), (4.28) we obtain for $d \in[0,1]$

$$
\left|\varepsilon_{i}^{*}\left(\beta^{*}, d, \varepsilon\right)\right| \leq c(|d|+|\varepsilon|+1) .
$$

With (2.12) and (4.24) this shows for $d \in[0,1]$

$$
\begin{aligned}
\left|\beta^{*} d_{1} d_{2} \operatorname{det}\left(\varepsilon_{2}^{*}(d, \varepsilon)-\varepsilon_{1}^{*}(d, \varepsilon)\right)\right| & \leq c\left(\left|\varepsilon_{1}^{*}(d, \varepsilon)\right|^{2}+\left|\varepsilon_{2}^{*}(d, \varepsilon)\right|^{2}+1\right) \\
& \leq c\left(|d|^{2}+|\varepsilon|^{2}+1\right) .
\end{aligned}
$$

From this we easily verify $(\mathcal{A} 2)_{1}$ restricted to $d \in[0,1]$, as the first two terms in (4.24) can be estimated as in the one-dimensional case.

In order to show $(\mathcal{A} 2)_{2}$, we apply (4.31) and (5.60) to find

$$
\begin{aligned}
\left|\bar{\sigma}^{*}(d, \varepsilon)\right| & =\left|\sum_{i=1}^{2} d_{i} \alpha_{i}\left(\varepsilon_{i}^{*}-\varepsilon_{i}^{T}\right)\right| \\
& \leq c(|d|+|\varepsilon|+1), \\
\left|\triangle \varepsilon^{*}(d, \varepsilon)\right| & =\left|\varepsilon_{2}^{*}-\varepsilon_{1}^{*}\right| \\
& \leq c(|d|+|\varepsilon|+1), \\
\left|\varphi\left(\triangle \varepsilon^{*}(d, \varepsilon)\right)\right| & =\left|\operatorname{det}\left(\triangle \varepsilon^{*}(d, \varepsilon)\right)\right| \leq\left|\varepsilon_{2}^{*}(d, \varepsilon)-\varepsilon_{1}^{*}(d, \varepsilon)\right|^{2} \\
& \leq c\left(|d|^{2}+|\varepsilon|^{2}+1\right) .
\end{aligned}
$$

Finally, from (4.32) and the fact that $T$ is an isometry w.r.t. the Frobenius norm,

$$
\left|\frac{\partial \beta^{*}}{\partial d}(d, \varepsilon)\right| \leq c
$$

The terms $W_{i}\left(\varepsilon_{i}^{*}(d, \varepsilon)\right), i=1,2$ remaining in (4.31) can be estimated as in the onedimensional case. This verifies $(\mathcal{A} 2)_{2}$ restricted to $d \in[0,1]$. 
Now we prove $(\mathcal{A} 2)_{3}$. For the regimes 0 , I and II this follows directly from (5.60). When in Regime III, we start from (4.30) and find with (5.62) for $d \in[0,1]$

$$
\left|d_{1}\left(\alpha_{2}-\gamma^{*} T\right) \alpha^{-1} \alpha_{1}\left(\varepsilon_{1}^{*}-\varepsilon_{1}^{T}\right)\right| \leq c\left(\left|\varepsilon_{1}^{*}\right|+1\right) \leq c(|d|+|\varepsilon|+1) .
$$

The validation of $(\mathcal{A} 1)$ relies on (4.29). By assumption (i) of the theorem, $\frac{\partial \beta^{*}}{\partial \varepsilon}=0$ and, as $d \in[0,1]$ is fixed in $(\mathcal{A} 1), \alpha^{-1}$ is a constant tensor. Assumption $(\mathcal{A} 1)$ then follows from

$$
\left(\varepsilon_{1}^{*}\left(d, \varepsilon_{2}\right)-\varepsilon_{1}^{*}\left(d, \varepsilon_{1}\right)\right):\left(\varepsilon_{2}-\varepsilon_{1}\right)=\alpha^{-1}\left(\alpha_{2}-\beta^{*} T\right)\left(\varepsilon_{2}-\varepsilon_{1}\right):\left(\varepsilon_{2}-\varepsilon_{1}\right)
$$

a similar equality for $\left(\varepsilon_{2}^{*}\left(d, \varepsilon_{2}\right)-\varepsilon_{2}^{*}\left(d, \varepsilon_{1}\right)\right):\left(\varepsilon_{2}-\varepsilon_{1}\right)$, and the positive definiteness of $\alpha^{-1}$ and $\left(\alpha_{i}-\beta^{*} T\right), i=1,2$. Indeed, the latter is equivalent to $\mathrm{Id}-\beta^{*} \alpha_{i}^{-1 / 2} T \alpha_{i}^{-1 / 2}$ positive definite, and since $\beta^{*} \leq \gamma^{*}$, this is implied by all eigenvalues of $\operatorname{Id}-\gamma^{*} \alpha_{i}^{-1 / 2} T \alpha_{i}^{-1 / 2}$ being positive. However, from (4.25) and the definition of $\gamma_{i}$, this is true.

For the scalar case in $D=3$, comparing (4.24) and (4.36) and since the partial derivatives coincide, the theory subsumes to the geometrically linear theory for $D=2$ and $\beta^{*}=$ 0 . Hence we can proceed as above and show that $\widehat{W}$ given by (4.36) satisfies $(\mathcal{A})$. The assumptions $(\mathcal{C} 1),(\mathcal{C} 2)$ are not needed here because $\beta^{*}=0$.

The following theorem states the uniqueness of weak solutions for the system with geometrically linear theory.

Theorem 6 (Uniqueness of weak solutions). Let $\mathcal{W}=\widehat{W}$ be given by 4.36 for $D=3$ (scalar setting), by (4.24) for $D=2$, or by $(4.47)$ for $D=1$. Assume further that $M \equiv 1$ and the elastic moduli of the two phases are equal, i.e. $\alpha_{1}=\alpha_{2}$. For $D=2$, let $\operatorname{det}\left(\varepsilon_{2}^{T}-\varepsilon_{1}^{T}\right) \leq 0$. Then the solution $(a, b, \mathbf{u})$ in Theorem 5 is unique in the spaces stated there.

Proof: (i) The case $D=1$.

For $\alpha_{1}=\alpha_{2}$, the equations (4.48), (4.49) read

$$
\varepsilon_{1}^{*}(d, \varepsilon)=\varepsilon+d_{2}\left(\varepsilon_{1}^{T}-\varepsilon_{2}^{T}\right), \quad \varepsilon_{2}^{*}(d, \varepsilon)=\varepsilon+d_{1}\left(\varepsilon_{2}^{T}-\varepsilon_{1}^{T}\right)
$$

which implies $\varepsilon_{2}^{*}-\varepsilon_{2}^{T}=\varepsilon_{1}^{*}-\varepsilon_{1}^{T}$ leading to $W_{1}\left(\varepsilon_{1}^{*}\right)-w_{1}=W_{2}\left(\varepsilon_{2}^{*}\right)-w_{2}$. This yields

$$
\widehat{W}(d, \varepsilon)=\frac{\alpha_{1}}{2}\left(\varepsilon_{1}^{*}-\varepsilon_{1}^{T}\right)^{2}+d_{1} w_{1}+d_{2} w_{2}=\frac{\alpha_{1}}{2}\left(\varepsilon+d_{1}\left(\varepsilon_{2}^{T}-\varepsilon_{1}^{T}\right)-\varepsilon_{2}^{T}\right)^{2}+d_{1} w_{1}+d_{2} w_{2} .
$$

In this special case, the partial derivatives of $\widehat{W}$ are

$$
\begin{aligned}
& \frac{\partial \widehat{W}}{\partial d}(d, \varepsilon)=\alpha_{1}\left(\varepsilon+d_{1}\left(\varepsilon_{2}^{T}-\varepsilon_{1}^{T}\right)-\varepsilon_{2}^{T}\right)\left(\varepsilon_{2}^{T}-\varepsilon_{1}^{T}\right)+w_{1}-w_{2}, \\
& \frac{\partial \widehat{W}}{\partial \varepsilon}(d, \varepsilon)=\alpha_{1}\left(\varepsilon+d_{1}\left(\varepsilon_{2}^{T}-\varepsilon_{1}^{T}\right)-\varepsilon_{2}^{T}\right) .
\end{aligned}
$$

Now we pass through the steps in the proof of Theorem 2 and make the necessary modifications. Let again $\left(a^{k}, b^{k}, u^{k}\right), k=1,2$ be two solutions to (1)-(3) and set $a:=a^{1}-a^{2}$, $b:=b^{1}-b^{2}, d^{k}:=a^{k}+b^{k}, \varepsilon^{k}:=\varepsilon\left(u^{k}\right)$ for $k=1,2$ and $d:=d^{1}-d^{2}=a+b, \varepsilon:=\varepsilon^{1}-\varepsilon^{2}$. 
The equations (3.20), (3.21) now read

$$
\begin{aligned}
& \int_{\Omega_{\mathcal{T}}}\left[\partial_{t} b \eta+\lambda \nabla b \cdot \nabla \eta+\alpha_{1}\left(\varepsilon+d\left(\varepsilon_{2}^{T}-\varepsilon_{1}^{T}\right)\right)\left(\varepsilon_{2}^{T}-\varepsilon_{1}^{T}\right) \eta\right] \mathrm{d} x \mathrm{~d} t \\
& \quad=\int_{\Omega_{\mathcal{T}}}\left[\frac{\vartheta}{2}\left(g^{\prime}\left(a^{2}+b^{2}\right)-g^{\prime}\left(a^{1}+b^{1}\right)+g^{\prime}\left(a^{1}-b^{1}\right)-g^{\prime}\left(a^{2}-b^{2}\right)\right) \eta+2 \kappa_{2} b \eta\right] \mathrm{d} x \mathrm{~d} t, \\
& \int_{\Omega_{t_{0}}} \alpha_{1}\left(\varepsilon+d\left(\varepsilon_{2}^{T}-\varepsilon_{1}^{T}\right)\right) \varepsilon(u) \mathrm{d} x \mathrm{~d} t=0 .
\end{aligned}
$$

Analogous to (3.23) we find

$$
\begin{aligned}
& \left.\frac{\lambda}{2}\left\|a\left(t_{0}\right)\right\|_{M}^{2}+\int_{\Omega_{t_{0}}} \lambda|\nabla a|^{2}+\alpha_{1}\left(\varepsilon+d\left(\varepsilon_{2}^{T}-\varepsilon_{1}^{T}\right)\right) a\left(\varepsilon_{2}^{T}-\varepsilon_{1}^{T}\right)\right) \mathrm{d} x \mathrm{~d} t \leq \\
& \quad \int_{\Omega_{t_{0}}} 2 \kappa_{1} a^{2}+\frac{\vartheta}{2}\left[\left|g^{\prime}\left(a^{1}+b^{1}\right)-g^{\prime}\left(a^{2}+b^{2}\right)\right|+\left|g^{\prime}\left(a^{1}-b^{1}\right)-g^{\prime}\left(a^{2}-b^{2}\right)\right|\right]|a| \mathrm{d} x \mathrm{~d} t .
\end{aligned}
$$

Choosing $\eta:=\left(b^{1}-b^{2}\right) \mathcal{X}_{\left(0, t_{0}\right)}$ in (5.63) and adding the resulting equation to (5.65), we obtain

$$
\begin{aligned}
& \frac{\lambda}{2}\left\|a\left(t_{0}\right)\right\|_{M}^{2}+\frac{1}{2}\left\|b\left(t_{0}\right)\right\|_{L^{2}}+\int_{\Omega_{t_{0}}}\left[\lambda\left(|\nabla a|^{2}+|\nabla b|^{2}\right)+\alpha_{1}\left(\varepsilon+d\left(\varepsilon_{2}^{T}-\varepsilon_{1}^{T}\right)\right)^{2}\right] \mathrm{d} x \mathrm{~d} t \\
& \leq \int_{\Omega_{t_{0}}} 2\left(\kappa_{1}|a|^{2}+\kappa_{2}|b|^{2}\right)+\int_{\Omega_{t_{0}}} \frac{\vartheta}{2}\left[\left|g^{\prime}\left(a^{1}+b^{1}\right)-g^{\prime}\left(a^{2}+b^{2}\right)\right|+\left|g^{\prime}\left(a^{1}-b^{1}\right)-g^{\prime}\left(a^{2}-b^{2}\right)\right|\right](|a|+|b|) \mathrm{d} x \mathrm{~d} t .
\end{aligned}
$$

It is noteworthy that we are only able to estimate $\int_{\Omega_{\mathcal{T}}} \alpha_{1}\left(\varepsilon+d\left(\varepsilon_{2}^{T}-\varepsilon_{1}^{T}\right)\right)^{2} \mathrm{~d} x \mathrm{~d} t$ which differs from the total mechanical energy $\int_{\Omega_{\mathcal{T}}} \widehat{W}(d, \varepsilon) \mathrm{d} x \mathrm{~d} t$.

With the Lipschitz continuity of $g^{\prime}$, and by applying the inequalities of Young and Gronwall to (5.66), we obtain as in the proof of Theorem 2

$$
\int_{\Omega_{\mathcal{T}}} \alpha_{1}(\varepsilon(u))^{2} \mathrm{~d} x \mathrm{~d} t=0 .
$$

With Korn's inequality, $u \equiv 0$ in $\Omega_{\mathcal{T}}$.

(ii) The scalar case in $D=3$.

By Theorem 3, for $\alpha_{1}=\alpha_{2}$,

$$
\begin{aligned}
& \nabla \eta_{1}^{*}(d, f)=f-d_{2}\left(f_{2}^{T}-f_{1}^{T}\right), \\
& \nabla \eta_{2}^{*}(d, f)=f+d_{1}\left(f_{2}^{T}-f_{1}^{T}\right) .
\end{aligned}
$$

Consequently, $\nabla \eta_{1}^{*}-f_{1}^{T}=\nabla \eta_{2}^{*}-f_{2}^{T}$ leading to $W_{1}\left(\nabla \eta_{1}^{*}\right)-w_{1}=W_{2}\left(\nabla \eta_{2}^{*}\right)-w_{2}$. Since $\beta^{*}=0$, the formula for $\widehat{W}$ has now the same structure as for $D=1$ and the proof follows exactly as in the first part (i).

(iii) The case $D=2$. 
By assumption, $\varphi(\beta, d, \varepsilon)>0$, which results in $\beta^{*} \equiv 0$ in $\Omega_{\mathcal{T}}$. In addition, for $\alpha_{1}=\alpha_{2}$, by Eqn. (4.28),

$$
\begin{aligned}
& \varepsilon_{1}^{*}(d, \varepsilon)=\alpha_{1}^{-1}\left(\left(\alpha_{1}-\beta^{*} T\right) \varepsilon-d_{2} \alpha_{1}\left(\varepsilon_{2}^{T}-\varepsilon_{1}^{T}\right)\right), \\
& \varepsilon_{2}^{*}(d, \varepsilon)=\alpha_{1}^{-1}\left(\left(\alpha_{1}-\beta^{*} T\right) \varepsilon+d_{1} \alpha_{1}\left(\varepsilon_{2}^{T}-\varepsilon_{1}^{T}\right)\right) .
\end{aligned}
$$

Consequently,

$$
\varphi(\beta, d, \varepsilon)=-\operatorname{det}\left(\left(\varepsilon_{2}^{*}-\varepsilon_{1}^{*}\right)(d, \varepsilon)\right)=-\operatorname{det}\left(\varepsilon_{2}^{T}-\varepsilon_{1}^{T}\right) .
$$

So again $\varepsilon_{1}^{*}-\varepsilon_{1}^{T}=\varepsilon_{2}^{*}-\varepsilon_{2}^{T}, W_{1}\left(\varepsilon_{1}^{*}\right)-w_{1}=W_{2}\left(\varepsilon_{2}^{*}\right)-w_{2}$, and the proof can be carried out as before.

\section{Conclusion}

In this article we derived extensions of the Allen-Cahn/Cahn-Hilliard system to elastic materials. In particular we included (i) the linear elastic energy derived by Eshelby, (ii) a geometrically linear theory of elasticity for $D \leq 2$, and (iii) a geometrically linear theory of elasticity for $D=3$ in the scalar setting. For all three cases we showed in a mathematically rigorous way the existence and the uniqueness of weak solutions, asserting the correctness of our approach. As a future goal, it is desirable to generalize the proof to the non-scalar, i.e. vectorial case in $D=3$ and to $D \geq 3$.

The generalized AC-CH models contain as special cases both the Allen-Cahn [9] and the Cahn-Hilliard equation [21] with linear elasticity. In our work the existence and uniqueness of weak solutions to the AC-CH model with linear elasticity follows in a straightforward way since Assumption $(\mathcal{A})$ required in the existence result Theorem 1 can easily be checked to hold true for the linear energy. This had not been shown before.

We point out that for the new cases (ii) and (iii), the formulas collected in Section 4 for the specific energy $\widehat{W}$ are essential for any numerical studies of the AC-CH models extended to microstructure. For related investigations and numerical methods we refer the interested reader to [7] and [8].

Besides the limiting assumption of constant temperature (cf. Introduction), the most important pending restriction is the postulation of small strain, included in (4). That is, it would be desirable to combine the AC-CH system with energies within the framework of the geometrically non-linear theory of elasticity. The technical problems of a large strain theory are striking, see, e.g., [4, 5, 15, 25], and it is in many cases not known how to compute explicit formulas for the relaxed energy functionals.

Similar mathematical difficulties arise when one wants to extend the AC-CH systems to elastic materials which are of polycrystalline structure. This is an open problem for future research. For related modelling aspects we refer to [10, for related mathematical aspects to [5, 6].

\section{Acknowledgment}

We have worked on this project while we were employed or were visitors at the Max Planck Institute for Mathematics in the Sciences, Leipzig; the Institute of Applied Mathematics, 
University of Bonn; and the Institute for Mathematics, University of Würzburg. Some part of this work was performed while TB was visiting the Hausdorff Research Institute for Mathematics, Bonn. We acknowledge the hospitality of all these institutions.

\section{References}

[1] Allen, S. M.; Cahn, J. W. Microscopic theory for antiphase boundary motion and its application to antiphase domain coarsening, Acta Metallurgica 1979, 27, 1085-1095.

[2] Ambrosio, L.; Fusco, N.; Pallara, D. Functions of bounded variation and free discontinuity problems, Clarendon Press, New York, 2000.

[3] Barrett, J. W.; Garcke, H.; Nürnberg, R. A phase field model for the electromigration of intergranular voids. Interfaces and Free Boundaries 2007, 9, 171-210.

[4] Bhattacharya, K. Comparison of the geometrically nonlinear and linear theories of martensitic transformation. Contin. Mech. Thermodyn. 1993, 5, 205-242.

[5] Bhattacharya, K.; Kohn, R. V. Elastic Energy Minimization and the Recoverable Strains of Polycrystalline Shape-Memory Materials. Arch. Rational Mech. Anal. 1997, 139, 99-180.

[6] Bhattacharya, K.; Schlömerkemper, A. Stess-induced phase transformations in shapememory polycrystals, Arch. Rational Mech. Anal. 2010, 196, 715-751.

[7] Blesgen, T.; Chenchiah, I. V. A generalized Cahn-Hilliard equation based on geometrically linear elasticity, Interfaces and Free Boundaries 2011, 13, 1-27.

[8] Blesgen, T. The elastic properties of single crystals with microstructure and applications to diffusion induced segregation. Crystal Research and Technology 2008, 43, 905-913.

[9] Blesgen, T.; Weikard, U. On the multicomponent Allen-Cahn equation for elastically stressed solids. Electr. J. Diff. Equ. 2005, 89, 1-17.

[10] Blesgen, T.; Schlömerkemper, A. Towards diffuse interface models with a nonlinear polycrystalline elastic energy. In: A. Doughett and P. Asnarez (Eds.), Composite Laminates: Properties, Performance and Applications, Nova Science Publishing 2010, pp. 465-489.

[11] Brochet, D.; Hilhorst, D.; Novick-Cohen, A. Finite-dimensional exponential attractor for a model for order-disorder and phase separation. Appl. Math. Lett. 1994, 7, 83-87.

[12] Cahn, J. W.; Hilliard, J. E. Free energy of a non-uniform system I. Interfacial free energy. J. Chem. Phys. 1958, 28, 258-267.

[13] Cahn, J. W.; Larché, F. C. The effect of self-stress on diffusion in solids. Acta Metall. 1982, 30, 1835-1845.

[14] Cahn, J. W.; Novick-Cohen, A. Evolution equations for phase separation and ordering in binary alloys. J. Stat. Phys. 1994, 76, 877-909. 
[15] Chenchiah, I. V.; Bhattacharya, K. The Relaxation of Two-well Energies with Possibly Unequal Moduli. Arch. Rational Mech. Anal. 2008, 187, 409-479.

[16] Cherkaev, A. V. Variational methods for structural optimization, Appl. Math. Sci. 2000, 140, Springer publishing.

[17] Cioranescu, D.; Donato, P. An introduction to homogenization, Oxford University Press, 1999.

[18] Dacorogna, B. Direct methods in the calculus of variations, Springer, Berlin, 1989.

[19] Eshelby, J. D. Elastic inclusions and inhomogeneities. Prog. Solid Mech. 1961, 2, 89140.

[20] Fratzl, P.; Penrose, O.; Lebowitz, J. L. Modelling of phase separation in alloys with coherent elastic misfit. J. Stat. Phys. 1999, 95, 1429-1503.

[21] Garcke, H. On Cahn-Hilliard systems with elasticity. Proc. Roy. Soc. Edinburgh Sec. A 2003, 133, 307-331.

[22] Gottstein, G. Physical Foundations of Materials Science. Springer Berlin, 2004.

[23] Kohn, R. V.; Vogelius, M. Relaxation of a variational method for impedance computed tomography. Comm. Pure Appl. Math. 1987, 60, 745-777.

[24] Kohn, R. V. The relaxation of a double-well energy. Continuum Mechanics and Thermodynamics 1991, 3(3), 193-236.

[25] Kohn, R. V.; Niethammer, B. Geometrically nonlinear shape-memory polycrystals made from a two-variant material. Math. Mod. Num. Anal. 2000, 34, 377-398.

[26] Milton, G.W. A brief review of the translation method for bounding effective elastic tensors of composites, Cont. Models and Discrete Systems (G.A. Maugin, ed.), Longman Scientific and Technical, 1990, 60-74.

[27] Novick-Cohen, A. Triple-junction motion for an Allen-Cahn/Cahn-Hilliard system. Physica D 2000, 137, 1-24.

[28] Nye, J. F. Physical properties of crystals: their representation by tensors and matrices, Clarendon Press; New York: Oxford University Press, 1984.

[29] Onuki, A. Ginzburg-Landau approach to elastic effects in the phase separation of solids. J. Phys. Soc. Japan 1989, 58, 3065-3068.

[30] Salje, E. Phase transitions in ferroelastic and co-elastic crystals. Cambridge University Press, 1990.

[31] Ziemer, W. Weakly differentiable functions: Sobolev Spaces and Functions of Bounded Variation, Graduate Texts in Mathematics, Springer, New York, 1989. 